\title{
Rho-A prenylation and signaling link epithelial homeostasis to intestinal inflammation
}

\author{
Rocío López-Posadas, ${ }^{1}$ Christoph Becker, ${ }^{1}$ Claudia Günther, ${ }^{1}$ Stefan Tenzer, ${ }^{2}$ Kerstin Amann, ${ }^{3}$ Ulrike Billmeier, ${ }^{1}$ Raja Atreya, ${ }^{1}$ \\ Gionata Fiorino, ${ }^{4}$ Stefania Vetrano, ${ }^{4}$ Silvio Danese, ${ }^{4}$ Arif B. Ekici, ${ }^{5}$ Stefan Wirtz, ${ }^{1}$ Veronika Thonn, ${ }^{1}$ Alastair J.M. Watson, ${ }^{6}$ \\ Cord Brakebusch, ${ }^{7}$ Martin Bergö, ${ }^{8}$ Markus F. Neurath, ${ }^{1}$ and Imke Atreya ${ }^{1}$ \\ ${ }^{1}$ Medical Clinic 1, University of Erlangen-Nuremberg, University Hospital of Erlangen, Erlangen, Germany. ${ }^{2}$ Institute of Immunology, University of Mainz, Mainz, Cermany. ${ }^{3}$ Department of Nephropathology, \\ University of Erlangen-Nuremberg, University Hospital of Erlangen, Erlangen, Germany. ${ }^{4}$ Humanitas Clinical and Research Center, Milan, Italy. ${ }^{5}$ Institute of Human Cenetics, \\ University of Erlangen-Nuremberg, University Hospital of Erlangen, Erlangen, Germany. ${ }^{6}$ Norwich Medical School, University of East Anglia, Norwich Research Park, Norwich, United Kingdom.

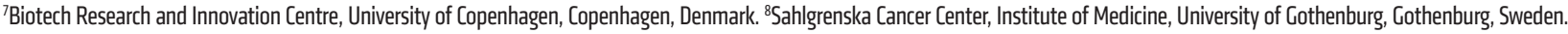

\begin{abstract}
Although defects in intestinal barrier function are a key pathogenic factor in patients with inflammatory bowel diseases (IBDs), the molecular pathways driving disease-specific alterations of intestinal epithelial cells (IECs) are largely unknown. Here, we addressed this issue by characterizing the transcriptome of IECs from IBD patients using a genome-wide approach. We observed disease-specific alterations in IECs with markedly impaired Rho-A signaling in active IBD patients. Localization of epithelial Rho-A was shifted to the cytosol in IBDs, and inflammation was associated with suppressed Rho-A activation due to reduced expression of the Rho-A prenylation enzyme geranylgeranyltransferase-I (CGTase-I). Functionally, we found that mice with conditional loss of Rhoa or the gene encoding CCTase-I, Pggt1b, in IECs exhibit spontaneous chronic intestinal inflammation with accumulation of granulocytes and CD4 ${ }^{+} \mathrm{T}$ cells. This phenotype was associated with cytoskeleton rearrangement and aberrant cell shedding, ultimately leading to loss of epithelial integrity and subsequent inflammation. These findings uncover deficient prenylation of Rho-A as a key player in the pathogenesis of IBDs. As therapeutic triggering of Rho-A signaling suppressed intestinal inflammation in mice with GGTase-I-deficient IECs, our findings suggest new avenues for treatment of epithelial injury and mucosal inflammation in IBD patients.
\end{abstract}

\section{Introduction}

Forming a mechanical boundary against pathogens, antigens, and toxins, the monolayer of intestinal epithelial cells (IECs) represents the human body's largest contact area with the environment (1). From their early development in the crypt bottom until shedding of aged cells at the villus tip, IECs follow a continuous turnover process (2-4). Under physiological conditions, a complex interaction between cytoskeleton rearrangement and tight junction proteins guarantees that the cell shedding itself does not mean a disturbance of epithelial integrity (5). However, alterations of epithelial integrity may lead to development of gut inflammatory disorders $(1,6)$, such as inflammatory bowel diseases (IBDs) (7). IBDs are associated with marked alterations of IECs, leading to increased tight junction permeability, altered cytoskeletal rearrangement, and induction of epithelial cell death with a subsequent loss of barrier function $(1,6)$. In vivo studies in IBD patients demonstrated that loss of IEC integrity even preceded the clinical relapse of disease (8), suggesting that alteration of barrier function is crucial in the pathogenesis of IBDs. However, the exact IEC intrinsic mechanisms involved in the initiation of barrier dysfunction remain enigmatic.

Authorship note: M.F. Neurath and I. Atreya contributed equally to this work. Conflict of interest: The authors have declared that no conflict of interest exists. Submitted: January 16, 2015; Accepted: November 18, 2015.

Reference information: / Clin Invest. 2016;126(2):611-626. doi:10.1172/JCI80997.
On a subcellular level, Rho GTPases represent signaling molecules centrally involved in arrangement of cytoskeletal proteins and epithelial cell dynamics (9-13). Since their activation preferentially takes place when GTPases are associated with cellular membranes $(14,15)$, function of Rho proteins crucially depends on their intracellular localization (16). Recruitment of small GTPases is governed by prenylation, a posttranslational process consisting of the attachment of hydrophobic isoprenoids to the C-terminal CAAX motif of the protein (17). In the intestinal epithelium, it has been shown that apoptotic as well as physiological cell extrusion requires Rho-mediated signaling pathways $(18,19)$. Moreover, Rho-A/ROCK/Myosin II interaction is known to be important for morphogenesis of the primitive gut tube in Xenopus (20). However, little is known about regulation of Rho-A signaling in the intestinal epithelium and its impact on gut homeostasis in vivo.

Using gene expression profiling of IECs from inflamed versus uninflamed tissue, we identified altered Rho-A signaling in IBD patients. Although Rho-A was expressed at normal levels, cytosolic accumulation of Rho-A in IECs suggested impaired Rho-A activation via prenylation upon inflammation. Consistently, genetic deletion of Rhoa or Pggt1b (encoding for the prenylationcatalyzing enzyme geranylgeranyltransferase-I [GGTase-I] driving Rho-A activation) in murine IECs impacted on cytoskeleton and cell shedding, resulting in epithelial injury and spontaneous gut inflammation. Our data demonstrate that injury in GGTase-I- 
A
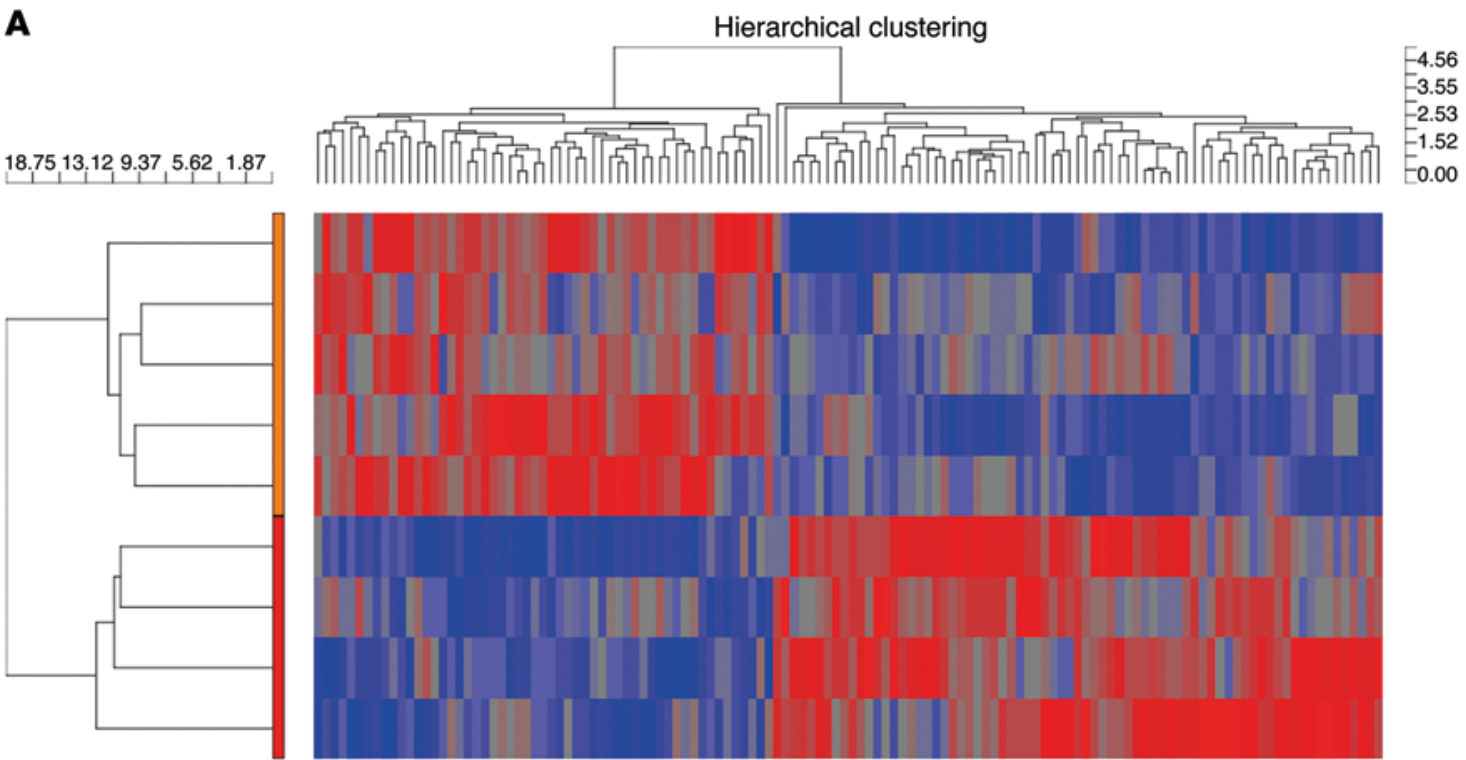

Phenotype $₫$ Uninflamed $\square$ Inflamed

$-222$

0.00

2.22

B

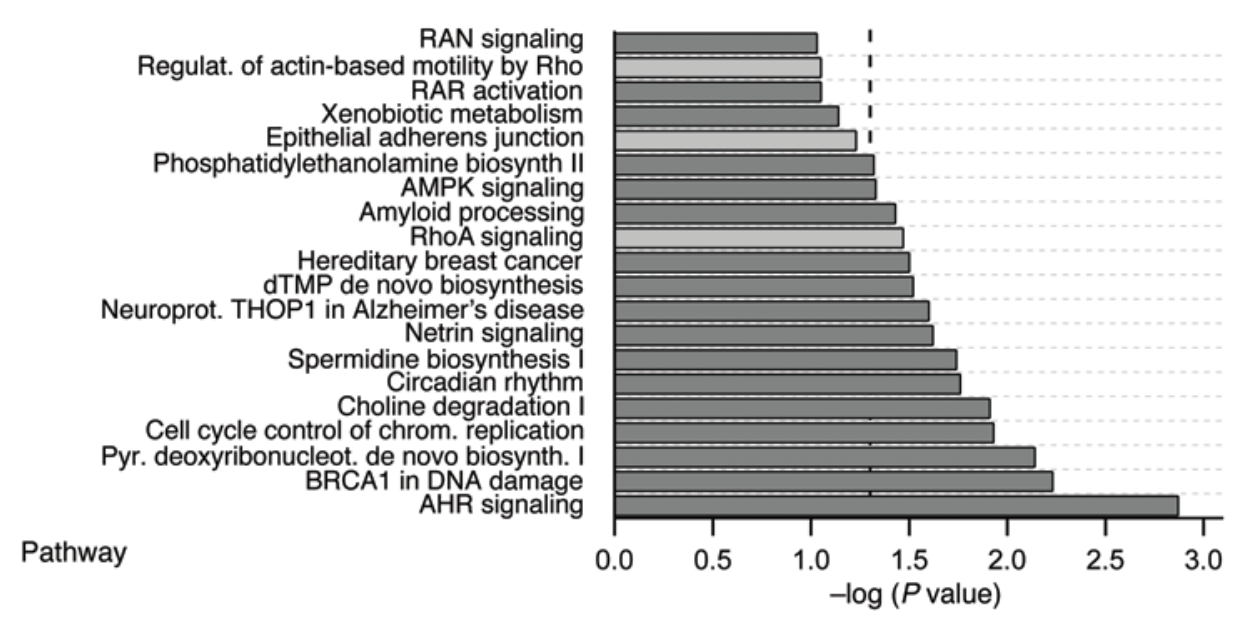

Figure 1. Gene expression array comparing IECs isolated from uninflamed and inflamed gut areas of CD patients. (A) Heat map showing differentially expressed genes in IECs (fold-change $\geq 1.5 ; P \leq 0.05$ ). (B) Top 20 regulated canonical pathways. Data are expressed as -log $(P$ value). $P$ value was obtained by independent sample $t$ test (Ingenuity analysis). $n=9$.

deficient epithelium was driven by Rho-A dysfunction and that this phenotype could be successfully reversed by Rho activation. Together, our study highlights for the first time to our knowledge the relevance of functional Rho-A signaling and its regulation via prenylation for maintenance of epithelial integrity. Thus, prenylated Rho-A in epithelium emerges as a therapeutic target in IBDs.

\section{Results}

A crucial role of epithelial Rho-A in intestinal inflammation. Intestinal erosions and ulcers caused by epithelial injury represent clinical hallmarks of IBDs, but the underlying pathogenesis of epithelial disruption in the context of gut inflammation is not fully understood. To identify alterations in signaling pathways in intestinal epithelium upon inflammation, we used a heuristic approach and performed gene expression arrays in purified IECs from Crohn's disease patients (CD patients) (array data accessible through NCBI's Gene Expression Omnibus [GEO GSE72780]). Clinical information of included patients is summarized in Supplemental Table 1 (supplemental material available online with this article; doi:10.1172/JCI80997DS1). Comparative genomic expression analysis of IECs from inflamed versus uninflamed gut areas of CD patients showed that samples within each group clustered, and we were able to identify 129 genes differing significantly (fold-change $\geq 1.5 ; P \leq 0.05$ ) between both groups (Figure $1 \mathrm{~A}$ ). Interestingly, gene ontology analyses indicated inflammation-associated downregulation of pathways involved in epithelial cell dynamics and IEC extrusion, such as Rho-A signaling, epithelial adherens junctions, and regulation of actin-based motility by Rho (Figure 1B). Notably, pathways involving Rho GTPases other than Rho-A, such as Cdc42 signaling, showed no significant changes (Supplemental Figure 1A). Although our data implicated a relation between impaired Rho-A function in IECs and inflammation in human IBDs, neither protein nor mRNA expression levels of Rho-A were found to be reduced in IECs from inflamed gut areas of CD or ulcerative 
A
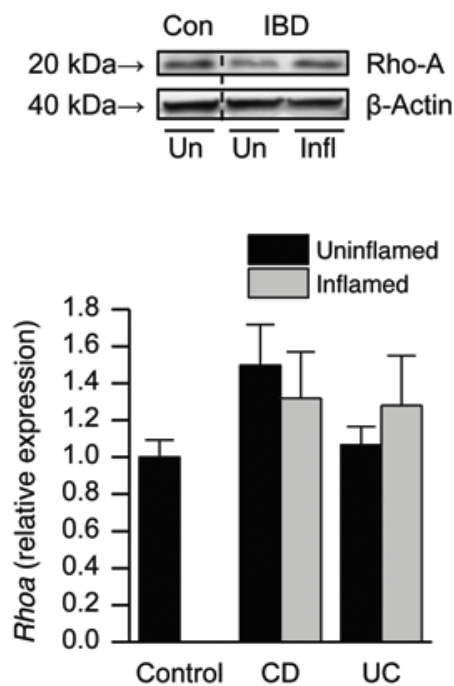

C

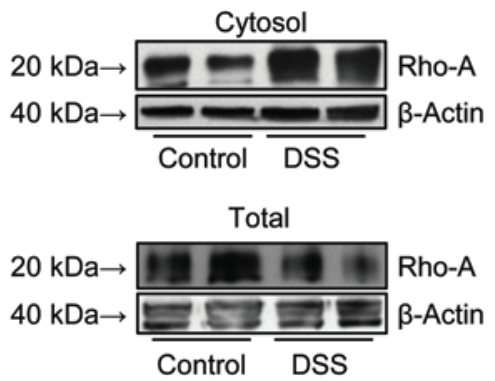

B
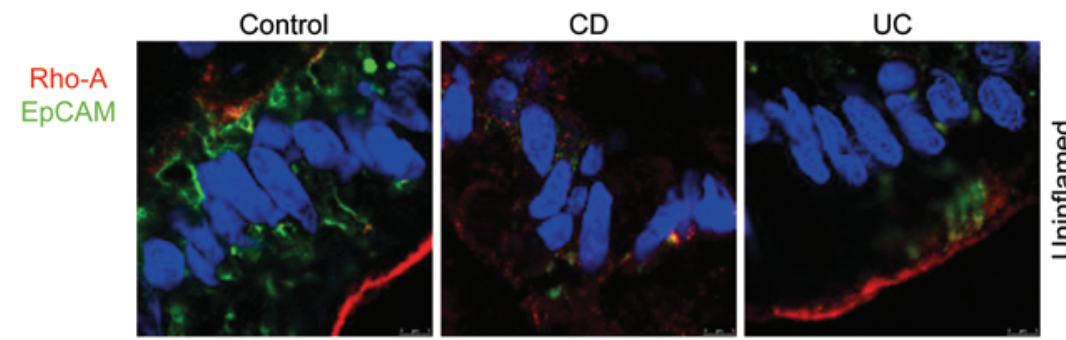

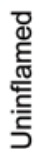

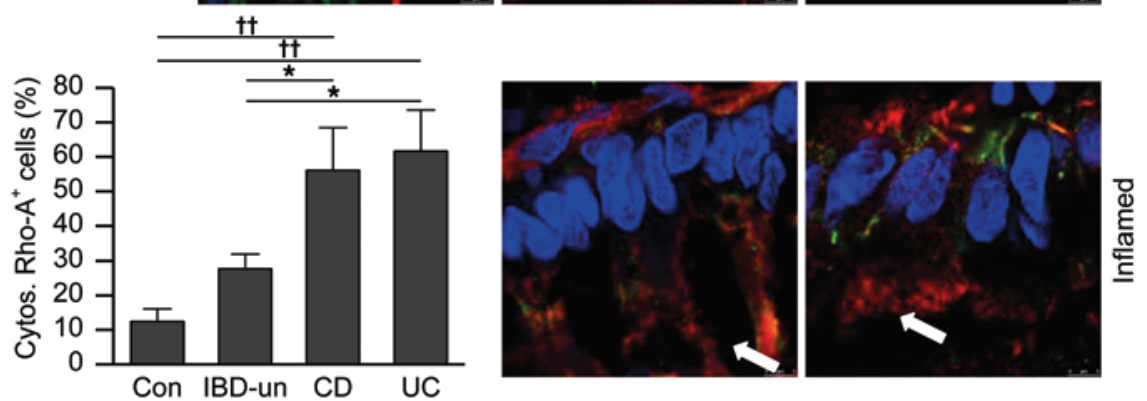

Figure 2. Rho-A profile in human and murine colitis. (A) Rho-A protein expression in IECs isolated from human gut; blots are representative of 2 experiments (top); and Rhoa mRNA expression (bottom) in IECs isolated from human gut. Mean values \pm SEM are shown (controls, $n=4$; IBD uninflamed, $n=9$; $\mathrm{CD}, n=5$; UC, $n=4$ ). No statistical significance, 1-way ANOVA with LSD multiple comparisons test. (B) Representative pictures and quantification from Rho-A immunostaining (red) in human gut samples. Sections were counterstained with EpCAM (green) and Hoechst (blue). Arrows indicate cytosolic accumulation of Rho-A. Bars show percentage of cells with cytosolic Rho-A (2 villi or crypts/sample; 30 IEC/sample). Mean values \pm SEM (controls, $n=5$; IBD uninflamed, $n=7 ; \mathrm{CD}, n=3$; UC, $n=3) .{ }^{\dagger+} P \leq 0.001 \mathrm{vs}$. control; ${ }^{*} P \leq 0.05 \mathrm{vs}$. IBD-uninflamed; 1 -way ANOVA with LSD multiple comparisons test. Original magnification, $\times 63$, zoom $\times 4$. (C) Western blot analysis of Rho-A in cytosolic and total proteins from colonic IECs from DSS-exposed and unchallenged mice. Blots are representative of 2 experiments ( $n=4 /$ group). Con, control; un, uninflamed; infl, inflamed.

colitis patients (UC patients) compared with uninflamed tissue (Figure 2A and Supplemental Table 2). However, while - in IECs of control patients - Rho-A was detected almost exclusively at the plasma membrane, IECs in inflamed areas of gut were characterized by a prominent cytosolic accumulation of Rho-A (Figure 2B, Supplemental Figure 1, B and C, and Supplemental Table 3), suggesting a predominance of inactive Rho-A in IECs of IBD patients. Similarly, cytosolic Rho-A enrichment upon inflammation could also be confirmed in IECs from dextran sulfate sodium-exposed (DSS-exposed) mice (Figure 2C and Supplemental Figure 1D), suggesting a link between dysfunction of epithelial Rho-A and gut inflammation. To further address the functional consequences of impaired epithelial Rho-A, we generated mice with an IEC-specific deletion of Rhoa (Rho-A ${ }^{\triangle I E C}$ mice) by crossbreeding mice carrying the LoxP-flanked Rhoa gene (21) with mice expressing Cre-recombinase under the control of the epithelium-specific villin promoter (22). Lack of Rho-A in IECs resulted in decreased body weight (Figure 3A) and spontaneous mucosal inflammation in the small intestine, as indicated by high resolution colonoscopy (Figure 3B). Evaluation of inflammatory tissue alteration by histologic scoring confirmed small intestinal pathology in Rho-A ${ }^{\triangle I E C}$ mice. In partic- ular, the small intestine of Rho- $\mathrm{A}^{\mathrm{\triangle IEC}}$ mice could be characterized by crypt abnormalities, villous blunting, reduced number of goblet cells (Figure 3, C and D, and Supplemental Figure 2A), marked infiltration of $\mathrm{CD}^{+} \mathrm{T}$ cells and neutrophils (Figure $3 \mathrm{E}$ and Supplemental Figure 2B), and significantly elevated levels of Tnfa (Figure 3F) compared with Rhoa ${ }^{\text {fl/ }}$ littermates. Colon tissue turned out to be less affected, and the increased numbers of $\mathrm{CD}^{+}{ }^{+} \mathrm{T}$ cells and increased mucosal TNF- $\alpha$ levels in the colon of Rho- $\mathrm{A}^{\mathrm{AEC}}$ mice did not reach statistical significance as compared with WT animals (Supplemental Figure 2, C and D). Interestingly, the observed intestinal phenotype of Rho- $\mathrm{A}^{\mathrm{AIC}}$ mice was not derived from a developmental problem during embryogenesis, since neonates from Rho-A $\mathrm{A}^{\triangle I E C}$ mice appeared to be healthy, showed no reduced body weight, and did not differ from Rhoa/f/f littermates with regard to intestinal tissue structure (Supplemental Figure 2, E-G). Our data therefore identified epithelial Rho-A as a key regulator in the maintenance of intestinal homeostasis and epithelial cell architecture. The altered epithelial structure in Rho-A ${ }^{\triangle I E C}$ mice was associated with a significantly increased intestinal permeability (Figure 3G), suggesting that epithelial Rho-A deficiency leads to impairment of intestinal barrier function. 
A

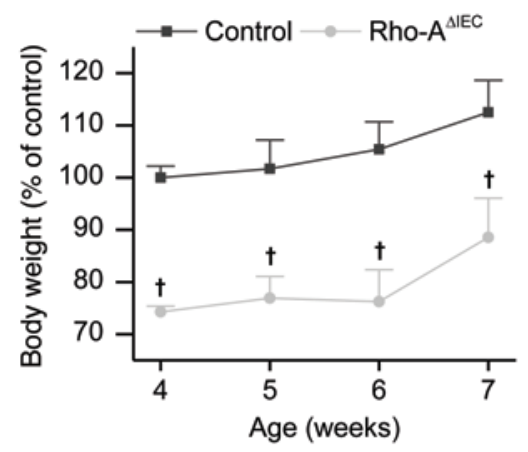

B

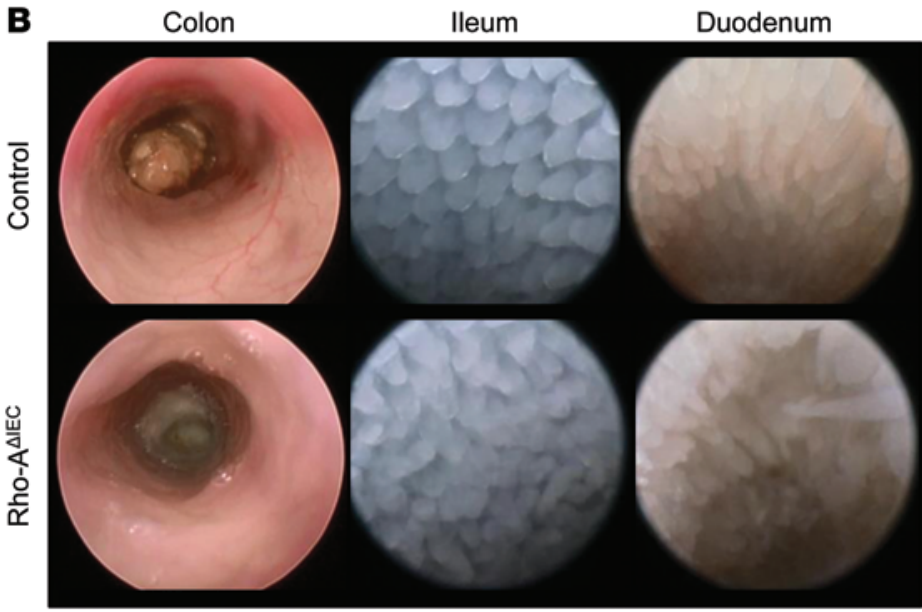

C
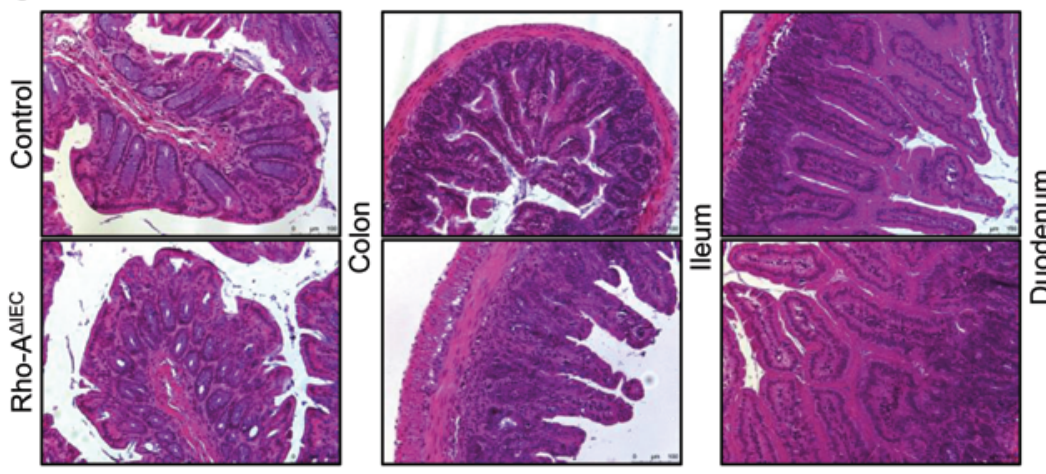

$\mathbf{F}$

E

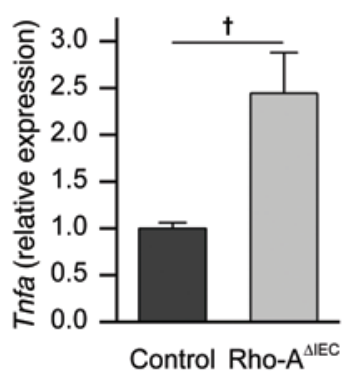

D

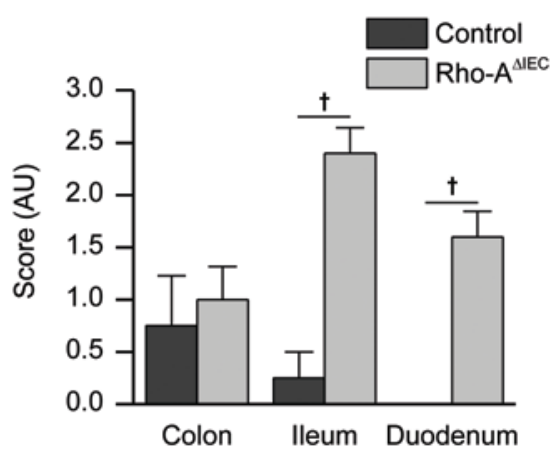

G

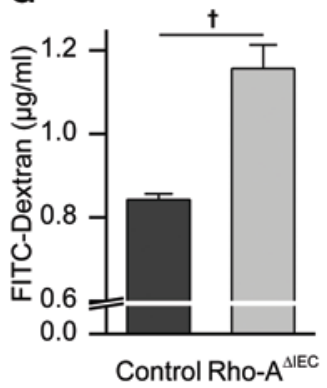

Figure 3. Phenotype of Rho-A ${ }^{\Delta E C}$ mice. (A) Body weight of control and Rho-A ${ }^{\Delta I E C}$ mice; percentage is calculated over the mean body weight of control mice on week 4. $(n=9)$. (B) Representative miniendoscopy pictures of different gut segments (3 experiments). (C) Representative H\&E pictures ( 3 experiments). Original magnification, $\times 20$. (D) Histological score quantification of different gut segments $(n=9)$. (E) Quantification of cell infiltration in ileum (immunofluorescence staining). $n=6$ /group. (F) Tnfa expression in ileum measured by qPCR. ( $n=6 /$ group). (G) Serum concentration of orally administered FITC-Dextran. $n=4$ /group. Data represent mean \pm SEM in $\mathbf{A}$ and $\mathbf{D}-\mathbf{G} .{ }^{\dagger} P \leq 0.05$ and ${ }^{\dagger \dagger} P \leq 0.001$ vs. control, independent samples $t$ test, in $\mathbf{A}, \mathbf{D}, \mathbf{F}$, and $\mathbf{G}$.

Deficient prenylation in the inflamed intestinal epithelium of $C D$ and UC patients. Since impaired Rho-A signaling in IBDs could not be explained by decreased Rho-A expression (Figure 2A), we postulated an impaired activation of epithelial Rho-A under inflammatory conditions via prenylation, a posttranslational modification necessary for membrane affinity of this GTPase (23). Notably, expression analysis of the Rho-A-associated prenylation-catalyzing enzyme GGTase-I indicated significantly diminished protein and mRNA levels in IECs of CD and UC patients during mucosal inflammation (Figure 4, A and B, Supplemental Figure 3A, and Supplemental Tables 3 and 4). The association between intestinal inflammation and diminished GGTase-I expression could be further confirmed in the experimental model of DSS-induced colitis in mice. In this model, significantly decreased GGTase-I expression went along with accumulation of nonprenylated $(\mathrm{Np})$ proteins in IECs upon inflammation, indicated by an increased detection of Np small GTPase Rap1A (Np-Rap1A) (Figure 4, C and D, and Supplemental Figure 3, B and C). Thus, deficient prenylation in IECs upon inflammation might be responsible for altered subcellular localization and signaling of Rho-A in IBDs.

To gain further insights into the signaling mechanisms controlling Pggt1b expression in gut epithelium, epithelial organoids were cultured in the presence of different proinflammatory cytokines. Use of gut organoid technique allowed us to perform our analyses in the absence of extraepithelial factors (24). Interestingly, exposure to the IBD hallmark cytokine IL-6 (25) resulted in a signifi- 
A
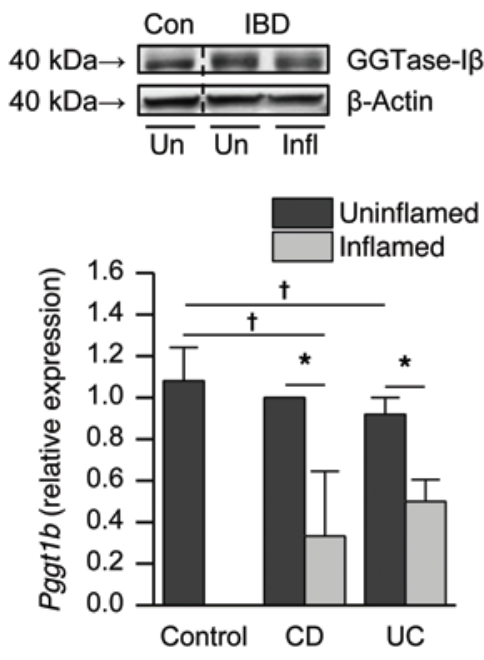

C
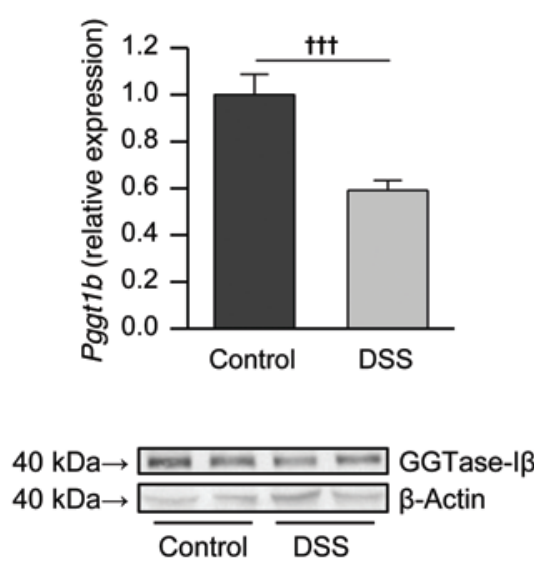

B
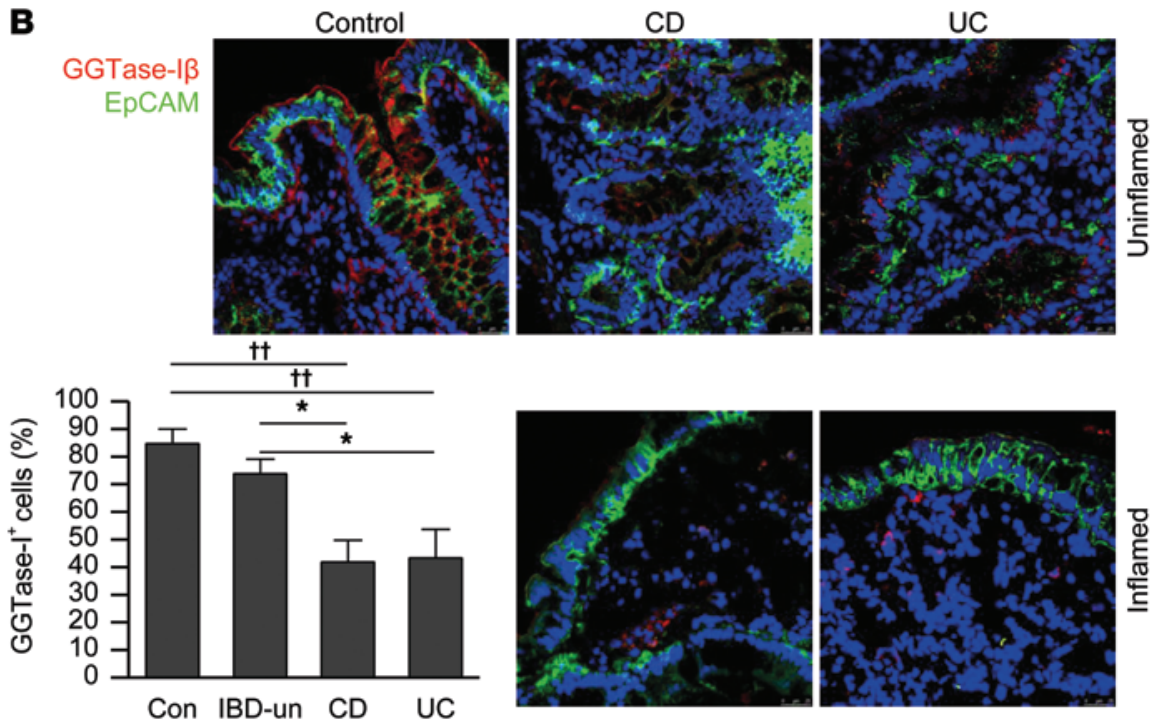

D

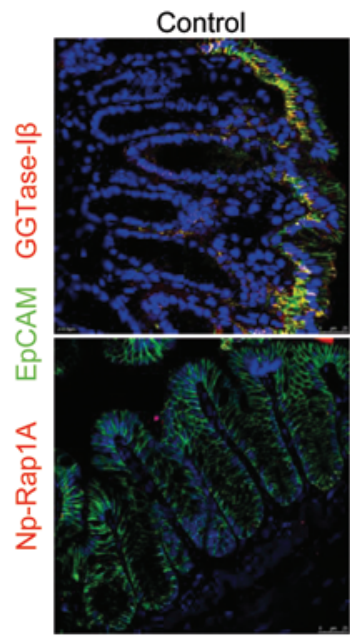

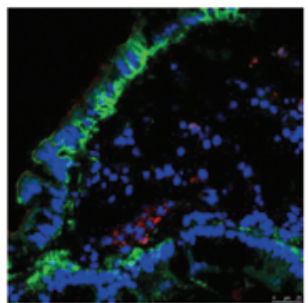

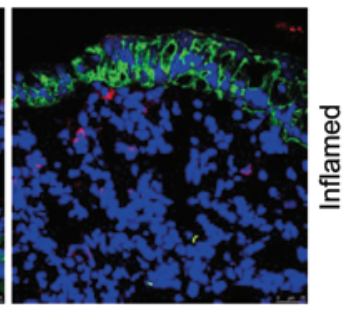

DSS
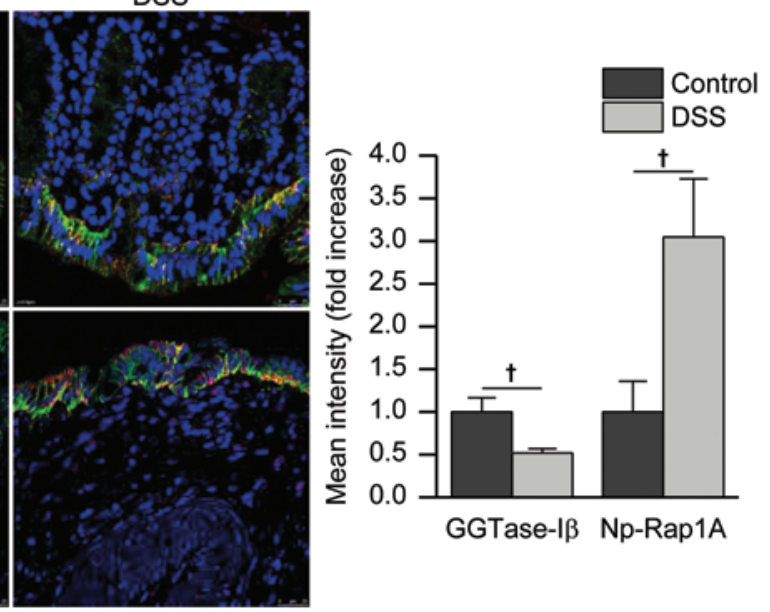

Figure 4. GGTase-I $\beta$ profile in human and murine colitis. (A) GGTase-I $\beta$ protein expression in IECs isolated from human gut; blots are representative of 2 experiments. The same samples are shown in Figure 2A (top); Pggt1b mRNA expression (bottom) in IECs isolated from human gut. (control, $n=3$; IBD uninflamed, $n=7$; CD, $n=3$; UC, $n=4$ ). ${ }^{\dagger} P \leq 0.05$ vs. control; ${ }^{*} P \leq 0.05$ vs. IBD uninflamed. (B) Representative pictures and quantification of GGTase-I $\beta$ immunostaining (red) in human gut samples. (control, $n=6$; IBD uninflamed, $n=7 ; C D, n=7 ; \mathrm{UC}, n=5$ ). Bars show percentage of cells expressing GCTase-I $\beta$ ( 2 villi or crypts/sample; $60 \mathrm{IEC} / \mathrm{sample).}{ }^{\dagger+} P \leq 0.001$ vs. control; ${ }^{*} P \leq 0.05$ vs. IBD uninflamed. Original magnification, $\times 63$. (C) GCTase-I $\beta$ expression in IECs from DSS-exposed mice: mRNA expression, measured by qPCR ( $n=8 /$ group). ${ }^{t+t} P \leq 0.0001 \mathrm{vs.} \mathrm{control} \mathrm{(top);} \mathrm{representative} \mathrm{blot} \mathrm{out} \mathrm{of} 3$ experiments (bottom). (D) GGTase-I $\beta$ immunostaining (red) in colon from DSS-treated mice. Mean intensity quantification ( $n=8 /$ group). Original magnification, $\times 63 .{ }^{\dagger} P \leq 0.05$ vs. control. Immunostainings were counterstained with EpCAM (green) and Hoechst (blue) in B and D. Mean values \pm SEM are shown in A-D. One-way ANOVA with LSD multiple comparisons test were used in A and B. Independent samples $t$ test was used in $\mathbf{C}$ and $\mathbf{D}$. Con, control; un, uninflamed; infl, inflamed.

cantly decreased Pggt1b expression in epithelial organoids (Figure $5 \mathrm{~A})$, suggesting that pathologically increased IL-6 signaling in the inflamed gut of IBD patients contributes to downregulation of Pggt1b expression and subsequent impairment of Rho-A activity in IECs. By comparing IECs in colon and small intestine, we observed contrary expression patterns for $I l 6$ and Pggt $1 b$ with high Il6 (Supplemental Figure 4A) and relatively low Pggt1b expression (Figure 5B) in the small intestine. Our data therefore implicate that regional as well as inflammation-induced alteration of prenylation levels in IECs might be augmented by IL-6. Besides relatively low levels of Pggt1b expression, IECs in small intestine could also be characterized by a decreased expression of Rhoa mRNA compared with IECs in colon (Figure 5B), making the small intestine potentially more susceptible to Rhoa and Pggt1b inhibition. As regional differenced in Rhoa expression could not be observed when we analyzed total gut tissue (Supplemental Figure 4B), our data furthermore suggest that regulation of intestinal Rhoa expression specifically occurs within the epithelial compartment.

Deficient prenylation within IECs results in alteration of cell shedding and loss of gut homeostasis. To further characterize the impact of prenylation on Rho-A signaling and intestinal inflammation in vivo, we created a mouse line with inducible conditional Pggt1b deficiency in IECs. Breeding of Pggt $1 b^{f l / f l}$ mice (26) with villin-Cre-ERT2 mice (22) (tamoxifen induces ERT2-dependent Cre-recombinase expression) led to the generation of Pggt-I ${ }^{\mathrm{Ti} i \mathrm{IEC}}$ mice, which developed normally and showed no signs of intestinal pathology before tamoxifen treatment (data not shown). Tamoxifen-induced specific deletion of Pggt1b within IECs caused 
A

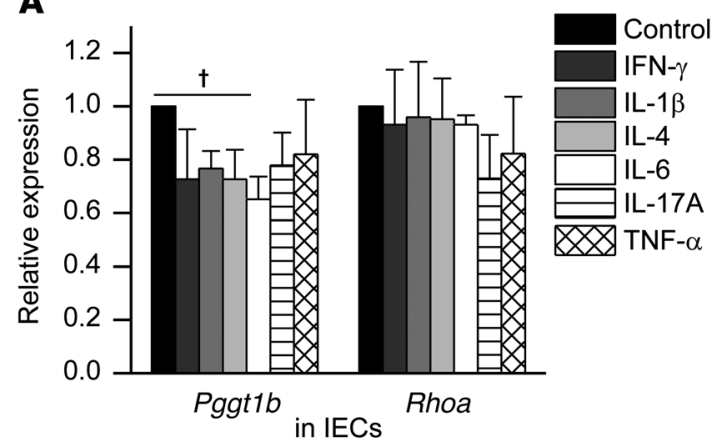

B

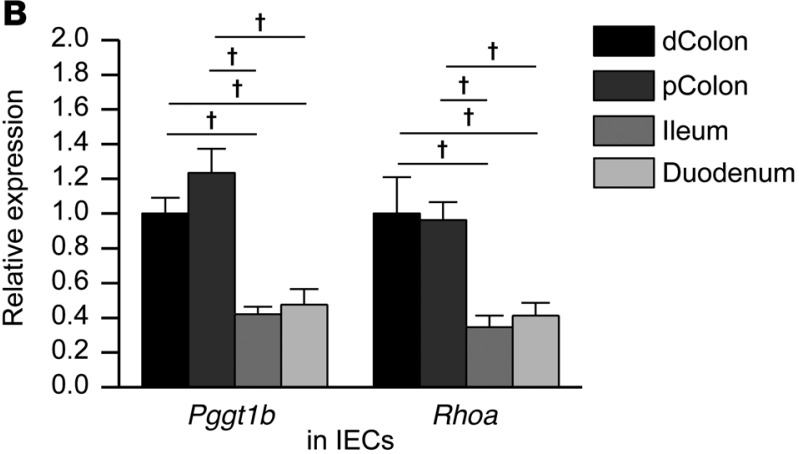

Figure 5. Regulation of GGTase-I $\boldsymbol{\beta}$ and Rho-A expression in intestinal epithelium. (A) Pggt1b and Rhoa mRNA expression (measured by qPCR) in cytokine-treated intestinal organoids from unchallenged WT mice (20 ng/ml of the cytokine, 8 hours stimulation) ( $n=3 /$ group). ${ }^{\dagger} P \leq 0.05$ vs. control; independent samples $t$ test. (B) Pggt1b and Rhoa mRNA expression in IECs isolated from different gut segments from unchallenged WT mice ( $n=4 /$ group). ${ }^{\dagger} P \leq 0.05$; 1-way ANOVA with LSD multiple comparisons test. Bars show mean $\pm \mathrm{SEM}$ in $\mathbf{A}$ and $\mathbf{B}$.

a decrease of epithelial Pggt1b mRNA (Supplemental Figure 5, A and B) and GGTase-I protein expression and, subsequently, an enrichment of Np proteins (Figure 6, A and B, and Supplemental Figure 5C). Such deletion resulted in body weight loss (Figure 7A), while Pggt $1 b^{f / f l}$ control mice remained unaffected. Macroscopic, endoscopic, and histological analyses identified chronic intestinal inflammation and injury in Pggt-I $\beta^{\mathrm{Ti} I \mathrm{IEC}}$ mice (Figure 7, B-E). The colon showed signs of moderate inflammation that did not reach statistical significance, whereas significant marked destruction of the villus-crypt architecture and loss of epithelial integrity could be observed in the small intestine of Pggt-I $\beta^{\text {TiAIEC }}$ mice as compared with floxed control mice (Figure 7E). Immunofluorescence and biochemistry analyses of small intestine tissue demonstrated that inflammatory injury of small intestine in Pggt-I $\beta^{\mathrm{TiAIEC}}$ mice went along with a massive infiltration of $\mathrm{CD} 4^{+} \mathrm{T}$ cells, macrophages, and neutrophils (Figure $7 \mathrm{~F}$ ) and significantly increased levels of proinflammatory cytokines, such as IL-1 $\beta$, IL-6, and TNF- $\alpha$ (Figure $7 \mathrm{G}$ and Supplemental Figure 5D). Colon tissue of Pggt-I $\beta^{\text {TiLIEC }}$ mice showed only moderate alterations with regard to inflammatory cell infiltration and cytokine production (Supplemental Figure 5, E an F). To investigate whether deficient prenylation in IECs might compromise the gut barrier, we next subjected mice to FITC-dextran oral gavage. We noted a significantly higher increase of FITC serum levels in Pggt-I $\beta^{\text {TiLIEC }}$ mice as compared with control mice, suggesting a breakdown of intestinal barrier function (Figure $7 \mathrm{H}$ ). Time course studies furthermore indicated that development of epithelial injury and impairment of gut barrier after Pggt1b deletion even preceded induction of proinflammatory cytokine production (Supplemental Figure 6). The loss of intestinal barrier function therefore appeared as a primary defect in Pggt-I $\beta^{\text {TiAIEC }}$ mice.

Figure 6. Validation of tamoxifen-induced Pggt1b deletion in IECs (Pggt-I $\boldsymbol{\beta}^{\text {TiIIEC }}$ mice). Pggt-I $\beta^{\text {TiLIEC }}$ mice were treated for 3 consecutive days with tamoxifen by i.p. injection. Day 0 was defined as the day of the first tamoxifen injection. (A) Western blot of GGTase-I $\beta$ and Np-Rap1A in isolated IECs; blots are representative of 3 independent experiments. (B) Representative pictures of GGTase-I $\beta$ (red) and Np-Rap1A (red) immunostainings in duodenum ( $n=4$ /group). Sections were counterstained with EpCAM (green) and Hoechst (blue). Original magnification, $\times 63$.
To investigate whether prenylation might be an essential process for epithelial cell survival, we next studied epithelial development in organoids (24) from Pggt-I $\beta^{\text {TiLIEC }}$ mice. Pggt $1 b$ deletion impaired the maintenance of complex epithelial organization and resulted in destruction and death of ex vivo cultured and tamoxifen-exposed organoids from Pggt-I $\beta^{\text {TiAIEC }}$ mice (Figure 8 and Supplemental Figure 7), suggesting that GGTase-I activity acts as an epithelial-intrinsic factor controlling cell survival. These data clearly demonstrated that gut epithelial organization and integrity directly depend on prenylation within IECs.

Dysregulation of apoptosis and necroptosis in IECs has been previously described to cause intestinal inflammation via loss of barrier function (4). To investigate whether excessive epithelial cell death might be a key driving factor of the severe phenotype occurring in Pggt-I $\beta^{\mathrm{Ti} \text { iLEC }}$ mice, we determined whether apoptosis or necroptosis might play an important role. However, neither the additional IEC-specific deletion of Casp8, nor that of the necrop-

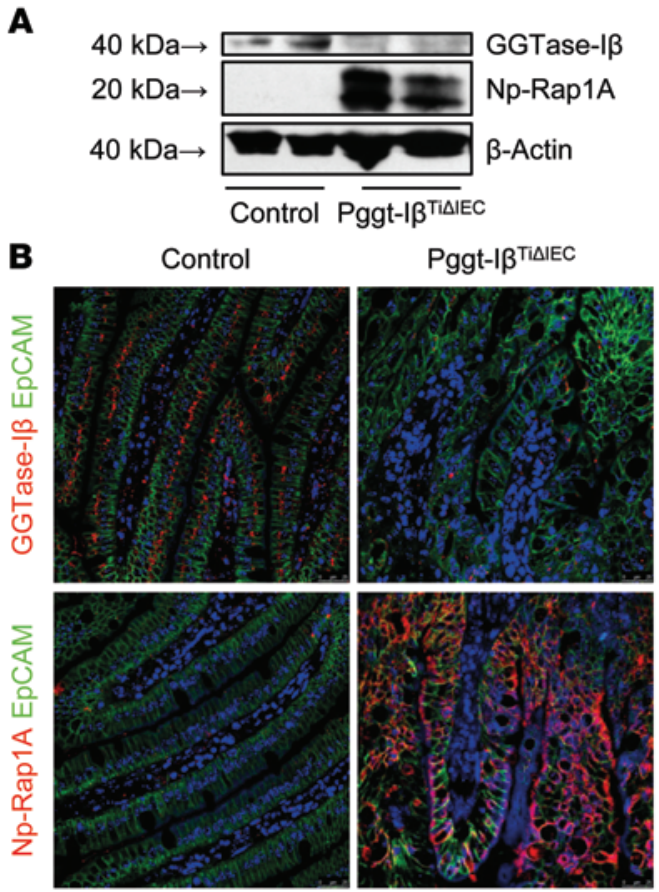


A

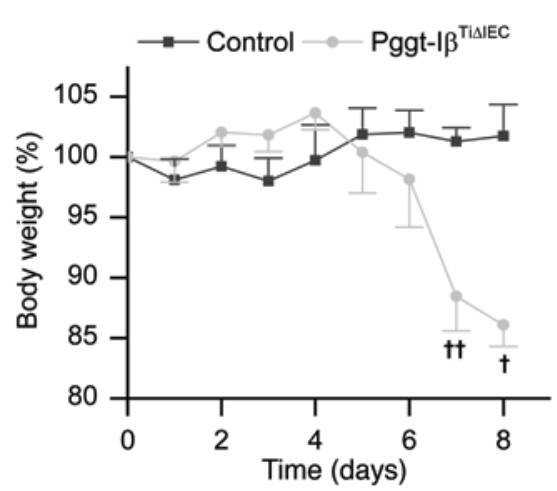

B

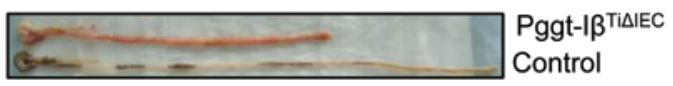

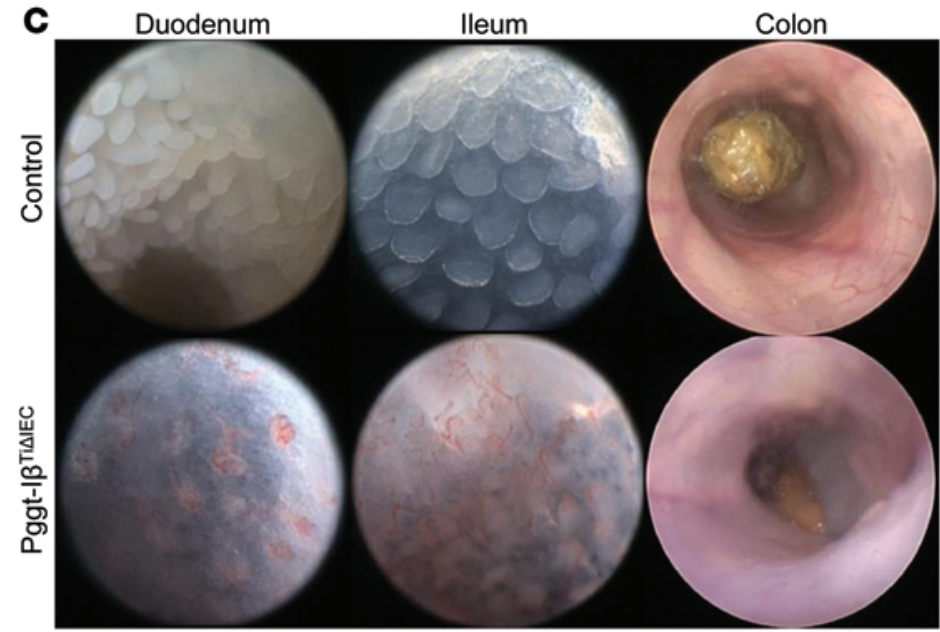

$\mathbf{E}$
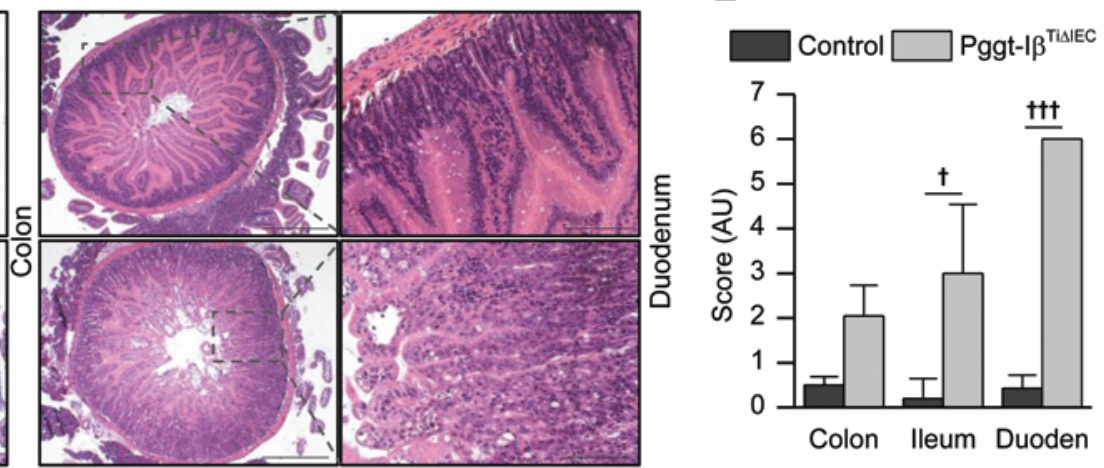

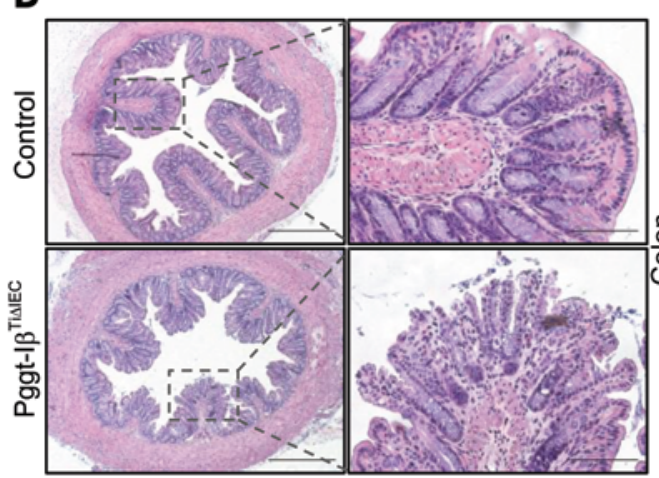

F $\square$ Control $\square$ Pggt-1 $\beta^{\text {TillEC }}$

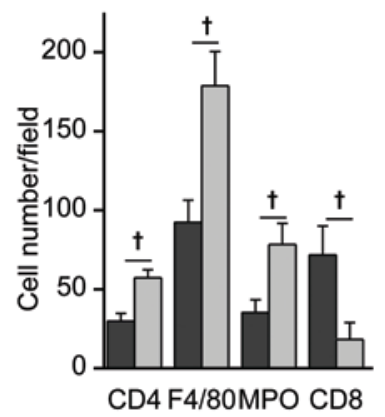

G

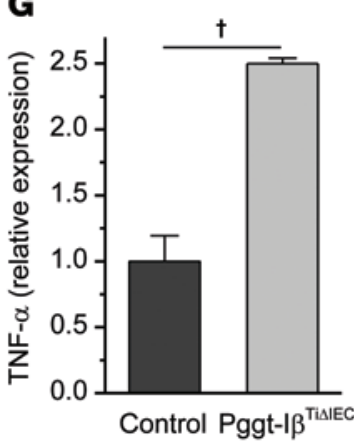

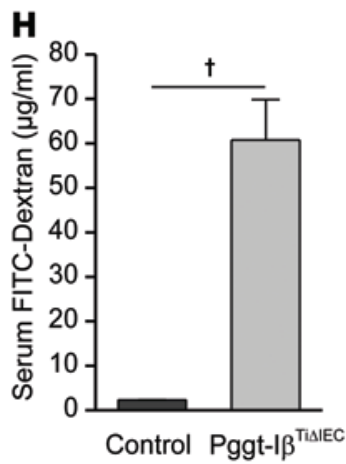

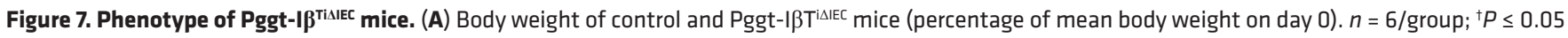
and ${ }^{t+} P \leq 0.001$ vs. control. (B) Macroscopic pictures of whole gut (3 experiments). (C) Mini-endoscopic pictures (3 experiments). (D) H\&E pictures. Original magnification, $\times 10$; insets, $\times 40$. (E) Histological score quantification of different gut segments ( $n=6 /$ group). ${ }^{\dagger} P \leq 0.05$ and ${ }^{t+t} P \leq 0.0001$ vs. control. (F) Quantification of cell infiltration in duodenum (immunofluorescence staining). $n=6$ /group. (C) TNF- $\alpha$ expression in duodenum measured by qPCR. $n=2 /$ group; representative of 3 experiments. (H) Serum concentration of orally administered FITC-Dextran $(n=4)$. Mean \pm SEM in $\mathbf{A}$ and $\mathbf{E}-\mathbf{H}$. ${ }^{\dagger} P \leq 0.05$ vs. control in $\mathbf{F}-\mathbf{H}$. Independent samples $t$ test, in $\mathbf{A}$ and $\mathbf{E}-\mathbf{H}$. Duoden, duodenum.

tosis inducer Rip3k, rescued the lethal consequence of Pggt1b deletion (Supplemental Figure 8, A-D). Additionally, proliferation of IECs along the crypt axis was not reduced in the absence of epithelial GGTase-I (Supplemental Figure 8E), excluding the possibility that cell proliferation arrest is a key underlying mechanism for tissue injury in Pggt-I $\beta^{\text {TiAIEC }}$ mice.

Our finding of severe barrier dysfunction in the absence of alterations in key homeostatic mechanisms finally led us to study epithelial cell shedding, a process crucial for the regulation of epithelial turnover in the steady state. Notably, using in vivo confocal microscopy of the gut (5), we observed marked alterations of the monolayer due to the occurrence of epithelial gaps in Pggt-I $\beta^{\text {TiaIEC }}$ mice but not control mice (Figure 9A). Under physiological conditions, such gaps are formed by the shedding of epithelial cells and sealed by neighboring cells $(3,5,27)$ to avoid breakdown of barrier function. To determine whether barrier function and cell shedding were altered in Pggt-I $\beta^{\text {TiAIEC }}$ mice, as recently observed in IBD patients $(8,28,29)$, we performed in vivo rhodamine leakage assays. These studies revealed an increased passage of topically applied dextran from thelumen to the lamina propriain Pggt-I $\mathrm{P}^{\mathrm{Ti \Lambda IEC}}$ 
A

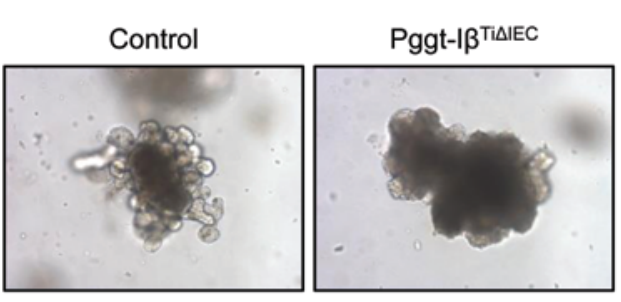

B

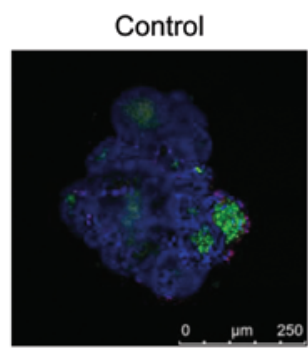

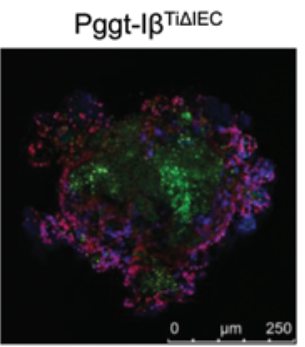

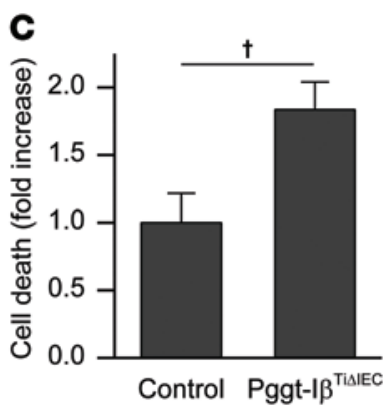

Figure 8. Development and survival of GGTase-I $\beta$-deficient organoids. Small intestinal crypts isolated from control and Pggt-I $\beta^{\text {TiAlEC }}$ mice were treated with tamoxifen in vitro. (A) Representative microscopic pictures out of 3 independent experiments. Original magnification, $\times 10$. (B and C) Cell death staining measured by propidium iodide incorporation (red) as described in Methods. Nuclei were counsterstained with Hoechst (blue). Representative pictures; original magnification, $\times 20(n=17)$ (B). Quantification of normalized mean fluorescence intensity (red/blue). Bars show mean values \pm SEM ( $n=17)$ (C).

${ }^{\dagger} P \leq 0.05$ vs. control; independent sample $t$ test.

as compared with control mice (Figure 9B and Supplemental Figure 9A). Villus tips in Pggt-I $\beta^{\mathrm{T} i \Delta \mathrm{IEC}}$ mice showed marked accumulation of leakage points and appearance of dextran-permeable IECs (Figure 9C), suggesting altered epithelial cell integrity. Furthermore, cell shedding was increased in Pggt-I $\beta^{\text {TisIEC }}$ mice (Supplemental Figure 9B), consistent with the barrier loss and suggesting shedding alterations as key processes mediating the phenotype of Pggt-I $\beta^{\text {TiLIEC }}$ mice.
Electron microscopic analysis of GGTase-I-deficient IECs revealed disruption of the epithelial architecture and altered cytoskeleton rearrangement (Figure 10A). Consistently, localization of actin fibers and myosin IIA within IECs was no longer restricted to the apical cell membrane in the absence of GGTase-I, but it spread along lateral membranes and also in the cytosol (Figure 10B and Supplemental Figure 10A). Cytoskeleton rearrangement was analyzed by kinome array of GGTase-I-deficient IECs.
A
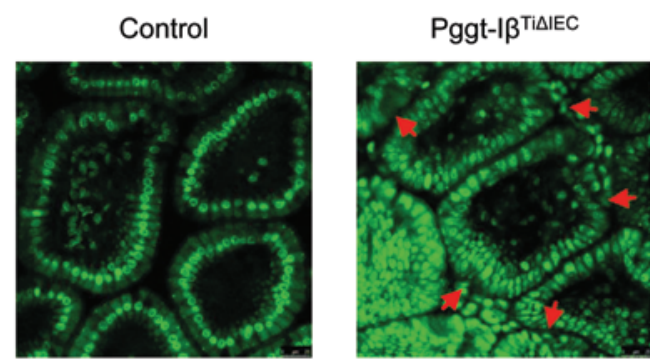

C
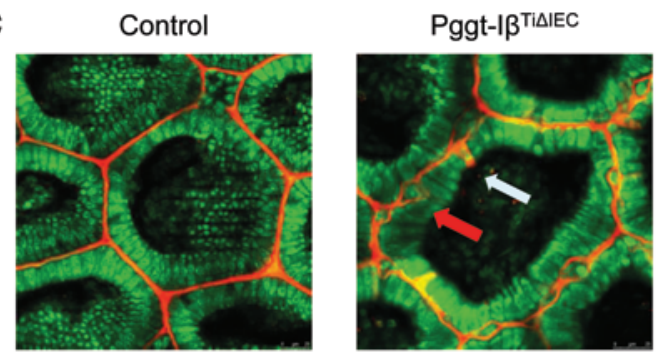

B

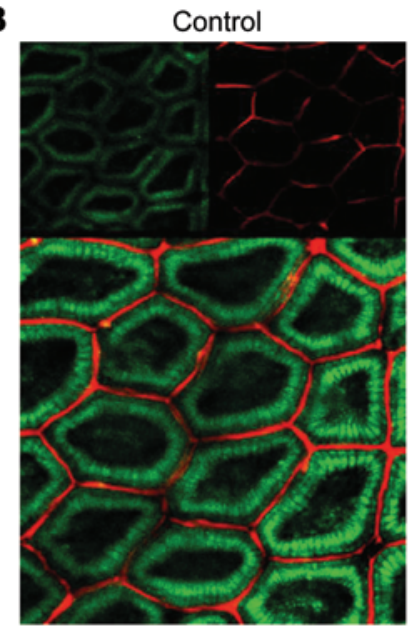

Pggt-I $\beta^{\text {TIIIEC }}$

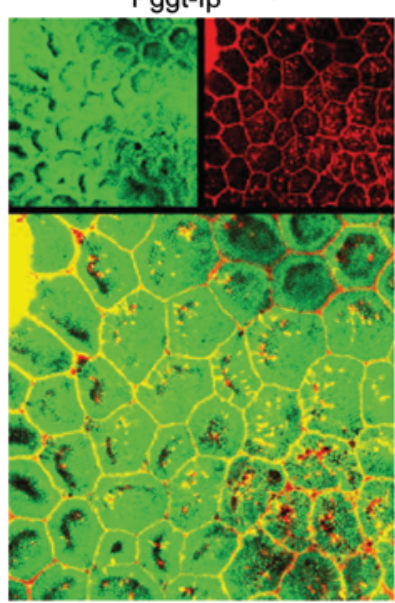

Leakage

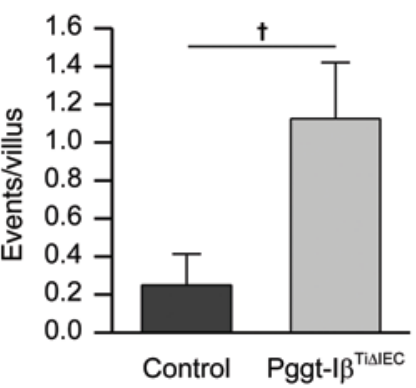

Permeable IECs

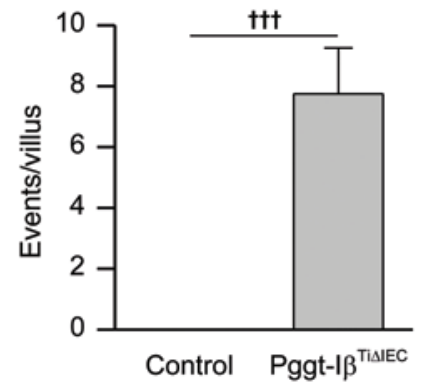

Figure 9. Epithelial turnover and integrity within GGTase-Iß-deficient epithelium. (A) Representative in vivo images of small intestinal villi stained with acriflavine (green) ( 3 independent experiments). Arrows indicate epithelial gaps. Original magnification, $\times 40$. (B) Live imaging of cell shedding in the small intestine from control and Pggt-I $\beta^{\text {TiLIEC }}$ mice; acriflavine (green), and luminal rhodamine-dextran (red). Representative pictures out of 2 experiments. Original magnification, $\times 20$. (C) Representative pictures and quantification of leakage entry points (red arrow) and permeable IECs (white arrow). Original magnification, $\times 40$. Mean $\pm \mathrm{SEM} ; n=8$ /group. ${ }^{\dagger} P \leq 0.05$ and ${ }^{\dagger+\dagger} P \leq 0.0001 \mathrm{vs}$. control mice; independent samples $t$ test. 
A
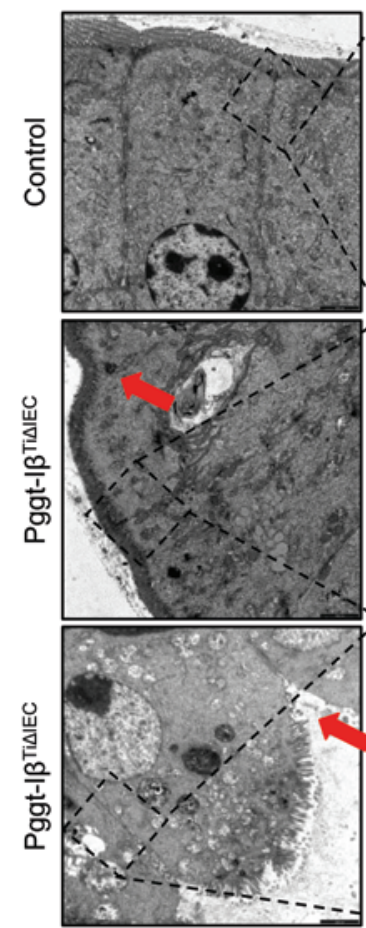
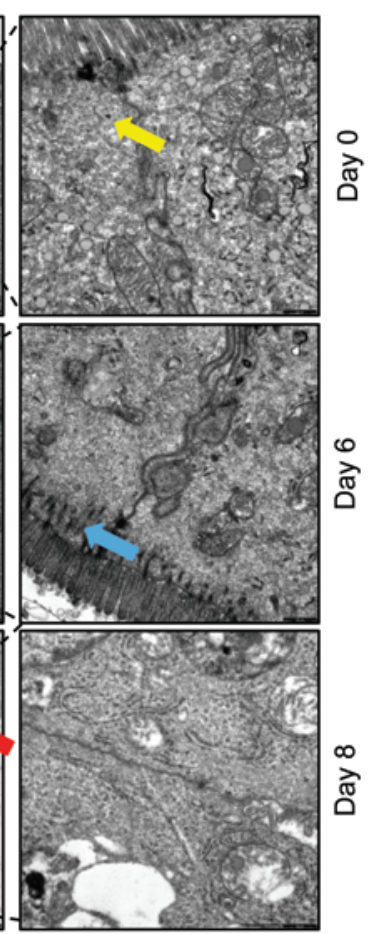

B
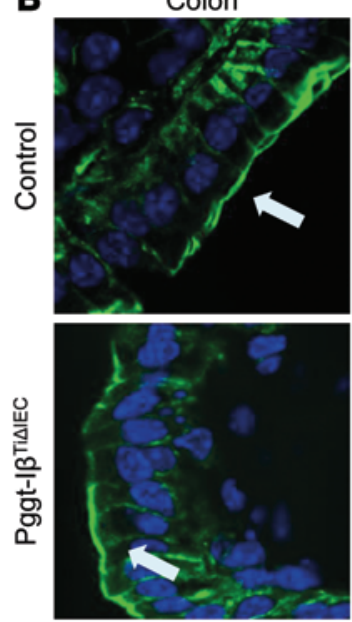

C
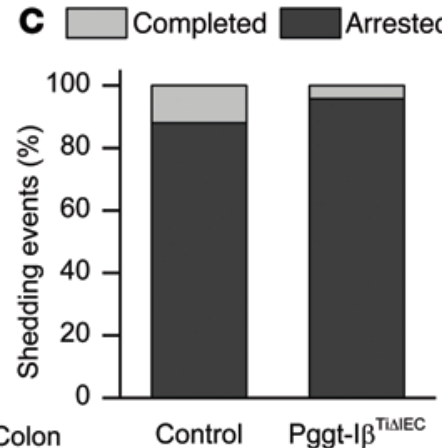

Duodenum
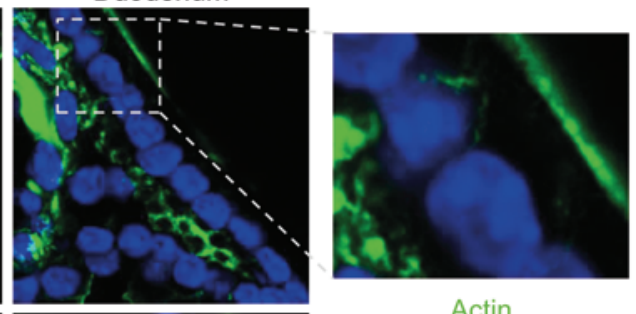

Actin
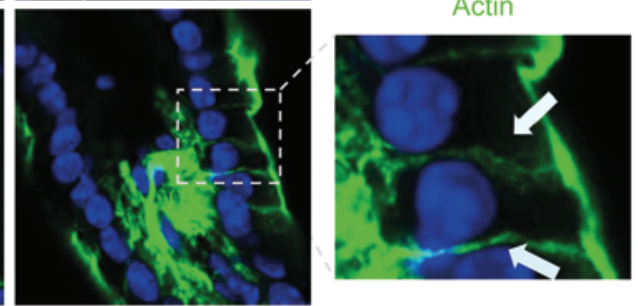

Completed

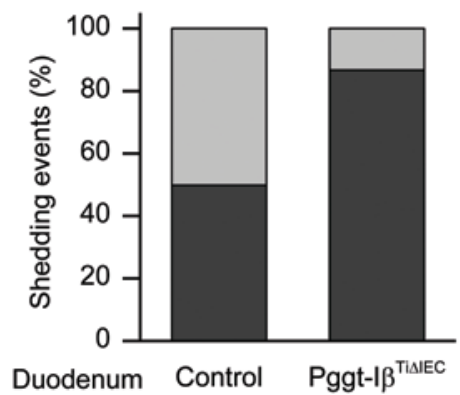

Figure 10. Cytoskeleton rearrangement and cell shedding within GGTase-Iß-deficient epithelium. (A) Representative electron microscopic pictures from duodenum of Pggt-I $\beta^{\mathrm{T} i \Delta \mathrm{EEC}}$ and control mice. Arrows indicate invaginations (red), apical network fibers (blue), and filamentous ultrastructure (yellow) (2 independent experiments). Original magnification, $\times 5,000$; insets $\times 20,000$. (B) Representative pictures from phalloidin staining of F-actin fibers (green) in colon and duodenum from Pggt- $\mid \beta^{\text {TiAlEC }}$ mice ( 3 independent experiments). Nuclei were counterstained with Hoechst. Arrows indicate redistribution of actin fibers. Original magnification, $\times 63$. (C) Quantification of arrested vs. completed shedding events, expressed as percentage of total shedding events. Mean values \pm SEM; $n=11$ (colon); $n=7$ (duodenum).

Phosphorylation of 19 out of 64 cytoskeleton-related proteins was modified, and many of these proteins are known to be involved in Rho-A regulation (Supplemental Figure 10B). Since cytoskeleton rearrangement and tight junction redistribution represent one of the early events occurring in cell shedding (27), we hypothesized that cell shedding was arrested in Pggt-I $\beta^{\text {TiAIEC }}$ mice. Hence, we quantified the number of IECs showing redistribution of actin fibers (funnel-like structures, arrested events) versus cells that have been already shed into the lumen (nucleus does not belong to the monolayer, completed events) (Supplemental Figure 10C). Indeed, the accumulation of arrested (early) versus completed (late) shedding events in GGTase-I $\beta$-deficient small intestine (Figure 10C) indicated an impaired completion of cell extrusion. Accumulated half-shed cells might subsequently impair the normal cytoskeletal mechanisms to seal the resulting gap.

Mucosal inflammation in the absence of GGTase-I is driven by blockade of Rho-A signaling. In order to further analyze the interplay between deficient prenylation, impaired Rho-A signaling, and epithelial injury, we next performed a more profound characterization of molecular alterations in GGTase-I $\beta$-deficient IECs. Quantitative proteomic and gene expression assays (array data accessible through NCBI's GEO [GSE72781]) revealed that the steady-state levels of 322 proteins and the expression of 725 genes were regulated in IECs of Pggt-I $\beta^{\text {TiAIEC }}$ mice as compared with control mice (fold-change $\geq 2 ; P \leq 0.05$ ) (Figure 11, A and B). Gene ontology analysis from GGTase-I-deficient IECs implicated an association between GGTase-I prenylation and cell viability/ cell death induction, cytoskeleton rearrangement, and cell morphology (Figure 11, C and D, and Supplemental Table 5), consistent with our mechanistic observations and previous findings on potential Rho targets (30). Interestingly, when we compared the gene expression profile in IECs from IBD patients and GGTase-Ideficient IECs, Rho-A signaling could be identified as one of the only 2 pathways significantly regulated in both conditions (Figure 11E). Beside Rho-A signaling, only the aryl hydrocarbon receptor (AHR) pathway (31) was regulated in both settings, but it seemed to be of minor functional relevance, since the expression of AHR repressor (AHRR) (32) turned out to be unchanged in IECs from Pggt-I $\beta^{\text {TiAIEC }}$ mice (Supplemental Figure 11). Furthermore, Rho-Aand GGTase-I-deficient IECs resembled each other in the accumulation of arrested cell shedding events (Figure 10, B and C, and Figure 12, A and B) and in their inability to form regular organoid structures (Figure 12C). Together, these data strongly suggested that impairment of Rho-A signaling represents the pivotal consequence of deficient GGTase-I activity in IECs, relevant for epithelial integrity and IBD pathogenesis. 
A

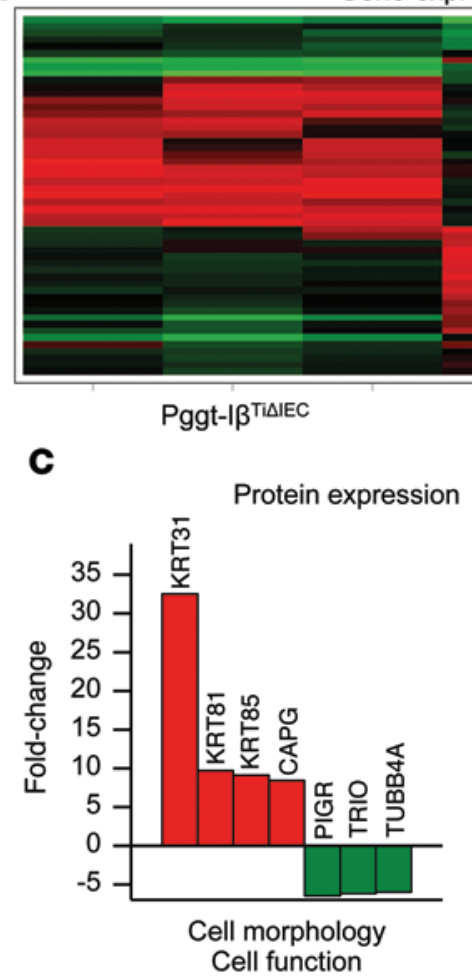

Gene expression

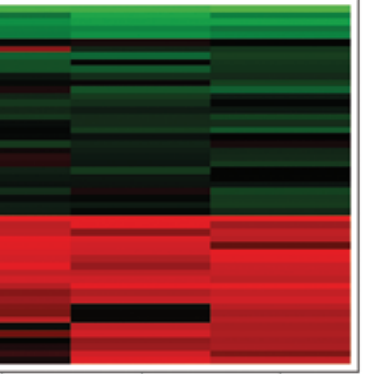

Control

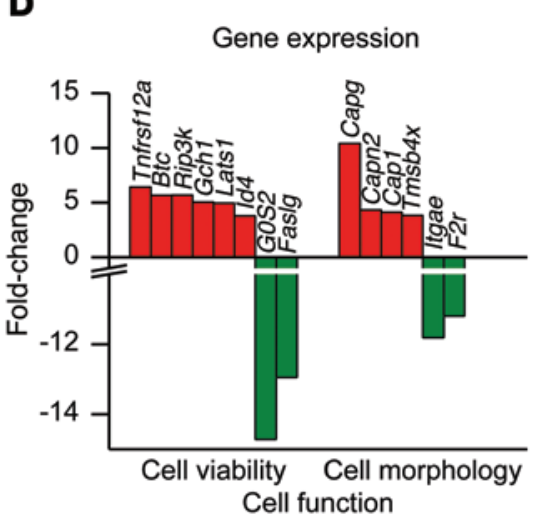

B
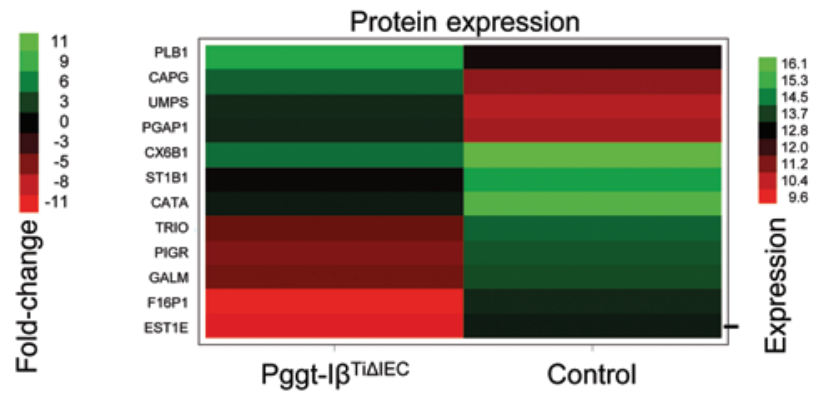

E

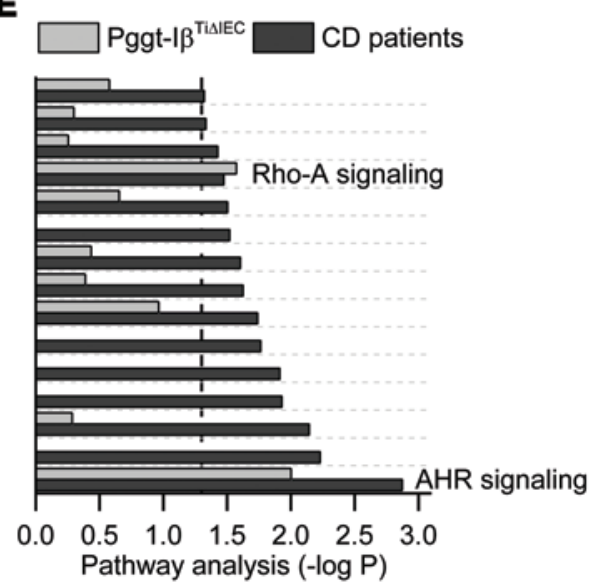

Figure 11. Molecular consequence of diminished protein prenylation in IECs. (A) Heat map of genes significantly regulated in IECs from Pggt-I $\beta^{\text {TiAIEC }}$ vs. control mice ( $n=3$ per group; fold-change $\geq 10 ; P \leq 0.05$; independent samples $t$ test). (B) Heat map of proteins significantly regulated in IECs from Pggt-I $\beta^{\text {TiLIEC }}$ vs. control mice ( $n=3$ for control and $n=2$ for Pggt-I $\beta^{\text {TiLIEC }}$ mice; fold-change $\geq 5 ; P \leq 0.05$; independent samples $t$ test). (C and $\left.\mathbf{D}\right)$ GO analysis. Representation of selected genes (C) and proteins (D) from Top 10 list clustered in groups related to their cellular function (fold-change). (E) Pathway analysis comparison between gene expression profiles in IECs from CD patients and Pggt-I $\beta^{\text {TiAlEC }}$ mice $(P \leq 0.05)$. Independent sample $t$ test.

In line with this suggestion, GGTase-I $\beta$-deficient IECs showed a substantial decrease of membrane-bound and an accumulation of cytosolic Rho-A (Figure 13, A and B, and Supplemental Figure 12A), while other small Rho GTPases did not show this profile (Supplemental Figure 12B). Reduced membrane-localization of Rho-A in IECs of Pggt-I $\beta^{\text {Ti } \triangle I E C}$ mice was paralleled by inefficient GTPase activation, resulting in reduced levels of active GTP-bound Rho-A (Figure 13C), and decreased phosphorylation of the downstream Rho-A target protein myosin light-chain 2 (MLC2) (Figure 13D). Thus, we confirmed that Rho-A activation and signaling is markedly impaired in IECs in the absence of GGTase-I. On a functional level, we wondered whether activation of Rho-A signaling would rescue the intestinal pathology in Pggt-I $\beta^{\text {TiLIEC }}$ mice and, thus, treated these mice with Rho activators. Analysis of Pggt-I $\beta^{\text {TiAIEC }}$ mice exposed to the specific Rho activator $\mathrm{CNO} 3$ indicated that successful triggering of Rho-A activity in IECs in vivo (Supplemental Figure 12C) resulted in a significant amelioration of mucosal damage in endoscopy (Figure 14, A and B), a clear tendency toward decreased histological damage score $(P=0.054)$ (Figure $14, C$ and $D)$, and significantly diminished expression of Illb and Il16 in the duodenum (Figure 14E) as compared with untreated mice. Rho-A blockade via impaired prenylation thus emerged as a key driver of mucosal damage in Pggt-I $\beta^{\mathrm{Ti} \Delta I E C}$ mice. The observed in vivo effect of $\mathrm{CNO} 3$ furthermore pointed to a potential therapeutic applicability of Rho activators for treatment of epithelial-derived gut pathology.

\section{Discussion}

Epithelial injury represents an important but not fully understood aspect of IBD pathogenesis (33). While recent studies have shown that epithelial injury in IBDs can be initiated by mechanisms regulating cell death in IECs, such as apoptosis and necroptosis (34), we here identified a new mechanism of epithelial damage in IBDs induced by impaired Rho-A signaling with subsequent cytoskeletal alterations of IECs and altered cell shedding. Such abnormal cell shedding may cause intestinal erosions and ulcer formation in IBDs and favors impaired barrier function that may clinically precede the development of acute flares in IBD patients $(8,27-29)$.

In the inflamed gut, epithelial-extrinsic mediators, such as proinflammatory cytokines derived from activated lamina propria immune cells (TNF- $\alpha$, IL-6, and IL-13) (35-37), are potent inducers of IEC apoptosis and pathological cell shedding with subsequent impairment of epithelial barrier function $(35,37-$ 40). However, an increased occurrence of abnormal intestinal permeability in healthy relatives from IBD patients (41), and the finding that loss of barrier function in IBDs often preceded the clinical relapse of disease $(8,28)$, strongly argue against the assumption that IBD-associated epithelial barrier defects rep- 

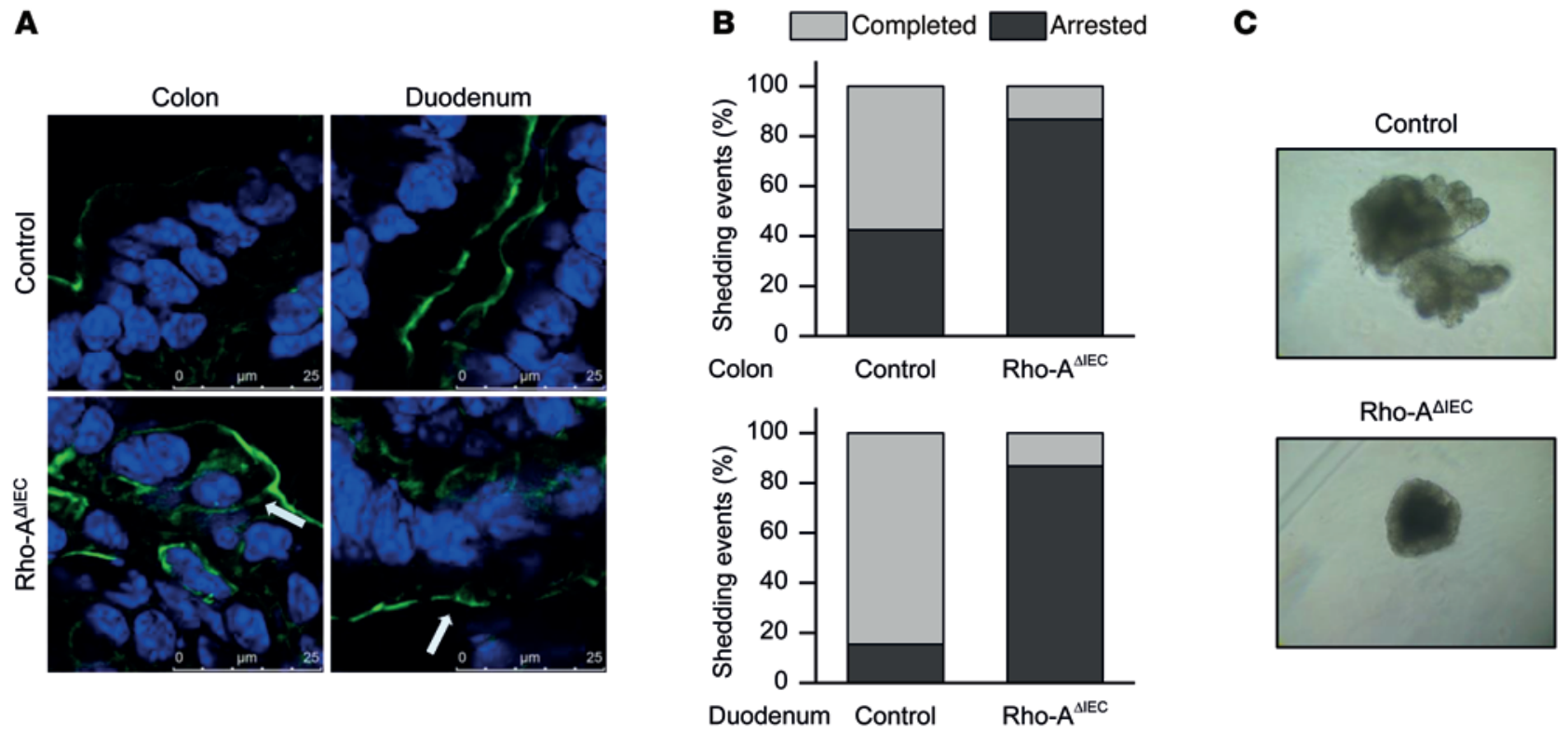

Rho-A $\triangle \mathrm{IEC}$

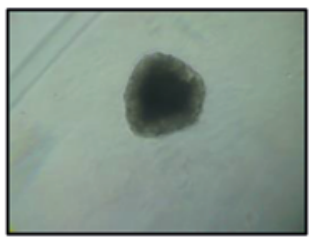

Figure 12. Epithelial integrity and turnover within Rho-A-deficient epithelium. (A) Cytoskeleton rearrangement within Rho-A-deficient IECs. Representative pictures from phalloidin staining of F-actin fibers (green) (3 experiments). Nuclei were counterstained with Hoechst. Arrows indicate redistribution of actin fibers. Original magnification, $\times 63$, zoom $\times 3$. (B) Quantification of arrested vs. completed shedding events in colon and duodenum, expressed as percentage of total shedding events. Mean values \pm SEM; $n=3$ per group. (C) Development of organoids generated from small intestinal crypts isolated from control and Rho-A $\mathrm{A}^{\Delta \mathrm{EC}}$ mice; representative pictures out of 2 experiments.

resent a pure secondary phenomenon of chronic colitis. Instead, epithelial-intrinsic alterations may play a primary role in IBD pathogenesis, and their modulation might open a field for potential therapeutic or preventive interventions. In this context, the here described requirement for sufficient Rho-A prenylation in gut epithelium represents an interesting new aspect, which significantly improves our understanding of intracellular regulation in IECs. In particular, the observation that Pggt1b deletion in ex vivo organoids impaired the maintenance of complex epithelial organization, even in the absence of any immune cell-derived factor, strongly supported the independency of Rho-A signaling cascades from epithelial-extrinsic mediators. On the other hand, the observed regulatory impact of IL- 6 on GGTase-I $\beta$ expression clearly demonstrated the high complexity of the interplay between epithelial and epithelial-extrinsic mediators in pathogenesis of IBDs. Our observation that IECs in small intestine of unchallenged WT mice showed as significantly decreased expression of Rhoa mRNA compared with IECs in colon might implicate augmented susceptibility of the small intestine to Rho-A and GGTase-I $\beta$ inhibition. It will therefore be important to investigate in future studies whether levels of epithelial Rho-A expression or activation along the gastrointestinal tract might indeed impact on regional differences in disease manifestations in IBD patients.

The functional relevance of Rho-A and GGTase-I-mediated Rho-A activation for functional integrity of the intestine was highlighted by studies in mice with conditional gene targeting in which deletion of Rhoa or Pggtlb in IECs caused spontaneous chronic intestinal inflammation with accumulation of granulocytes and $\mathrm{CD}^{+} \mathrm{T}$ cells and augmented production of the proinflammatory cytokine TNF- $\alpha$. The phenotype of these mice was particularly prominent in the small intestine, while little inflammation was noted in the large intestine. This could be due to the compensatory effects of other small GTPases in the large intestine. The identification of these GTPases will require further studies in the future.

Our findings indicate that epithelial integrity, intestinal architecture, and homeostasis critically depend on sufficient prenylation of Rho proteins within IECs. IEC-specific deletion of the enzyme GGTase-I resulted in intestinal disease characterized by accumulation of inactive cytosolic Rho-A, epithelial injury, and mucosal inflammation. Likewise, abrogation of Rho-A in IECs led to marked epithelial alterations and intestinal inflammation. Our findings suggested that impairment of GGTase-I-mediated prenylation in IECs causes accumulation of Np cytosolic Rho-A molecules that are functionally inactive. This subsequently results in impaired regulation of cytoskeleton rearrangement (42) with instability of cell shape and alteration of cell shedding. Accumulation of arrested cell shedding events within the epithelial monolayer finally resulted in loss of barrier function and pathologically increased intestinal permeability. Altered intestinal permeability may subsequently cause chronic intestinal inflammation. Indeed, time-course studies in inducible GGTase-I-deficient mice revealed that altered barrier function precedes mucosal inflammation, suggesting a primary barrier defect in such animals. The potential relevance of these findings for humans was underlined by the observation that significant downregulation of GGTase-I levels and cytosolic Rho-A accumulation was seen in the gut epithelium of IBD patients with active disease. Thus, in addition to the role of Rho-A signaling in cytoskeleton regulation (43), maintenance of cell shape (44), and cell migration (45), Rho-A emerges as a crucial regulator of gut epithelial homeostasis, and this function critically depends on cellular availability of prenylated Rho-A. Our findings indicated that Rho-A signaling is involved in epithelial cell shedding during inflammation. Consistently, inhibi- 
A

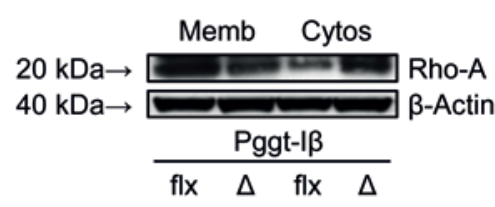

C

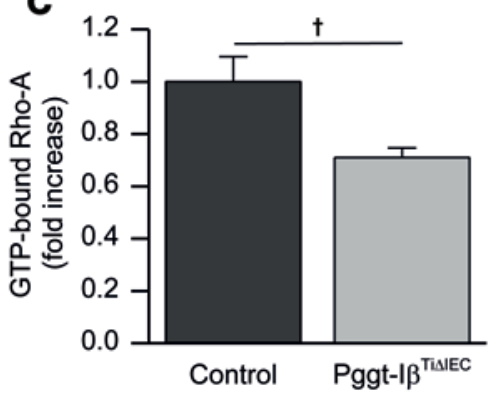

B

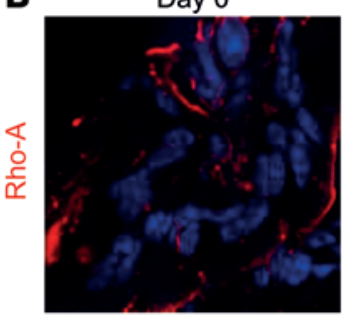

D
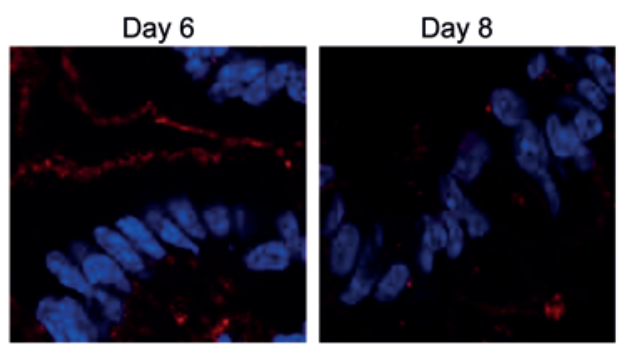

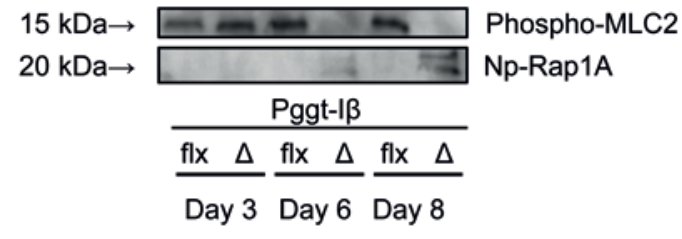

Figure 13. Rho-A dysfunction in Pggt-I $\boldsymbol{\beta}^{\mathrm{Ti} A \mathrm{IEC}}$ mice. (A) Western blot of Rho-A in membrane-bound and cytosolic proteins from IECs of Pggt-I ${ }^{\mathrm{Ti} i \mid \mathrm{E} C}$ and control mice; blots are representative of 3 experiments. (B) Representative Rho-A immunostaining (red) in colon from Pggt-I $\beta^{\text {TiliEC }}$ mice ( 2 independent experiments). Nuclei were counterstained with Hoechst. Original magnification, $\times 63$. (C) GTP-bound Rho-A in IECs from control and Pggt-I $\beta^{\text {TiAlEC }}$ mice. Mean values \pm SEM; $n=5$ samples/group; ${ }^{\dagger} P \leq 0.05$ vs. control; independent samples $t$ test. (D) Western blot of phosphorylated MLC- 2 and Np-Rap1A in IECs from control and Pggt-I $\beta^{\text {TiAIEC }}$ mice; blots are representative of 2 experiments.

tion of the Rho-associated kinase (ROCK), as well as deletion of the Rho-A target MLC kinase (MLCK), has been shown to result in incomplete cell extrusion (27). Microscopically, ROCK and MLCK inhibition resulted in a predominant appearance of funnel-like structures (arrested shedding events) built up by reorganized actin filaments and tight junction proteins along lateral membranes of shedding cells. However, the final consequence of the accumulation of arrested shedding events has not been considered previously (27). Such accumulation of epithelial funnel-like structures could also be observed in the intestine of Pggt-I $\beta^{\text {TiNIEC }}$ mice, indicating that redistribution of tight junction proteins takes place, but complete extrusion and resolution of shedding does not occur. Altered cell shedding in the absence of appropriate Rho-A function finally resulted in epithelial instability and loss of barrier function, leading to chronic intestinal inflammation. Hence, our observations confirmed that blockade of cell shedding due to cytoskeleton alterations, mediated by Rho-A among others, is an essential mechanism for disturbances of epithelial integrity.

The newly gained insights into Rho-A-dependent IEC intrinsic mechanisms involved in the initiation of intestinal barrier dysfunction open new avenues for potential interventional or translational approaches. Therapeutically triggered induction of Rho-A signaling via specific Rho activators or induction of Rho downstream signaling molecules such as ROCK might therefore represent a novel strategy for maintenance of intestinal integrity and prevention or healing of mucosal damage in IBDs and other inflammatory disorders of the intestine.

\section{Methods}

Animal models. Mice carrying LoxP-flanked Pggt1b (Pggt1 $\left.b^{f / f f}\right)$ (26), caspase $8\left(\operatorname{Casp}^{f l / f l}\right)(4)$, Rhoa alleles $\left(R h o a^{f / f f}\right)(21)$, as well as Rip3k KO mice (46), were described previously. Pggt $1 b^{f / f l}$ mice were provided by Martin
O. Bergö (Sahlgrenska Cancer Center, Institute of Medicine, University of Gothenburg, Gothenburg, Sweden). Rhoa ${ }^{f / l}$ mice were provided by Cord Brakebusch (Biotech Research and Innovation Centre, University of Copenhagen, Copenhagen, Denmark). To generate specific deletion of Pggt1b or Rhoa genes in IECs, Pggt $1 b^{f / f l}$ or Rhoa $a^{f / f l}$ mice were crossbred with villin-Cre or villin-Cre-ERT2 mice (22). Pggt-I $\beta^{\text {TiAIEC }}$ mice were crossbred with Casp $8^{f / f l}$ or Rip3 $k^{-/}$in order to impair apoptosis or necroptosis, respectively. Ethanolic tamoxifen (Sigma-Aldrich) solution was emulsified in sunflower oil and administered for 3 consecutive days by i.p. or intrarectal routes $(1 \mathrm{mg} / \mathrm{mouse} /$ day). Littermates carrying loxpflanked target genes but not the Cre-recombinase were used as control. Genotyping was performed by PCR in ear or tail genomic DNA. Animal studies were conducted in a sex- and age-matched manner using littermates for each experiment. Both male and female animals were used at the age of 6-10 weeks. All mice were kept under specific pathogenfree conditions. Except for DSS-induced colitis, animals from different experimental groups were cohoused in every experiment. Mice were routinely screened for pathogens according to Federation for Laboratory Animal Science Associations (FELASA) guidelines.

DSS-induced colitis. Colitis was induced in C57BL/6J mice by daily supplementation of drinking water with $2 \% \mathrm{w} / \mathrm{v}$ dextran sulfate sodium (MP Biomedicals) for 7 days $(47,48)$; DSS was withdrawn on day 7 , when animals received normal drinking water. Animals were sacrificed 10 days after starting of the DSS treatment.

Mouse endoscopy. Gut status of mice was monitored by high-resolution mouse video endoscopy as previously described (49). Mice were anesthetized with $2 \%-2.5 \%$ isoflurane in oxygen during endoscopy. Any colitis was scored basing on the following 5 parameters: thickening of the colon wall, changes in the normal vascular pattern, presence of fibrin, mucosal granularity, and stool consistency. Endoscopic scoring of each parameter was performed ( 0 to 3 ), leading to a cumulative score between 0 (no signs of inflammation) and 15 (very severe inflammation) (50). 
A

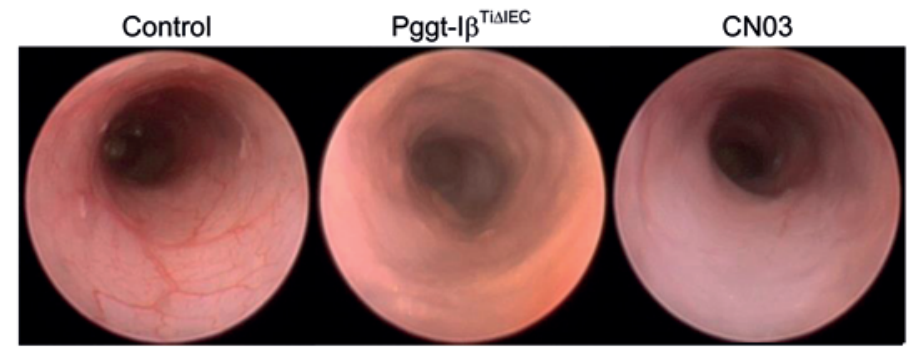

C

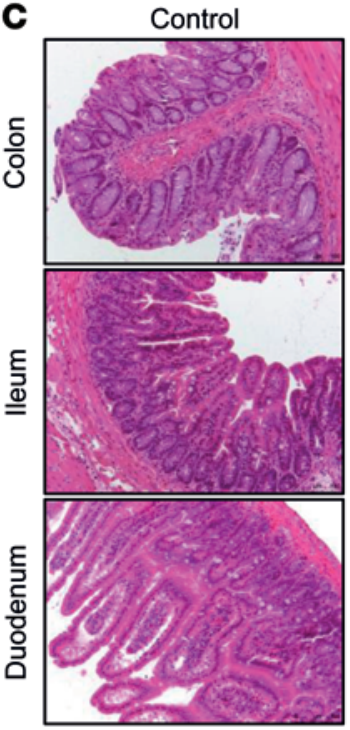

E
Pggt-I $\beta^{\text {TIIIEC }}$
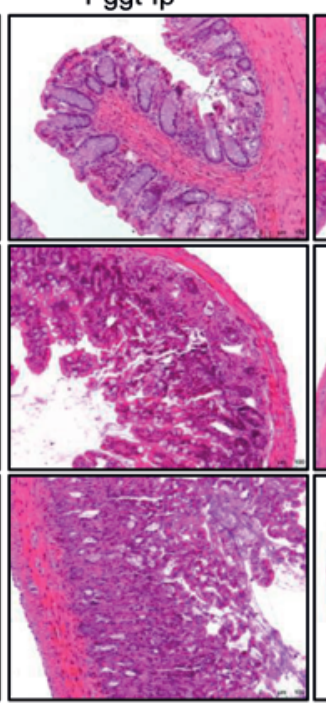

CNO3
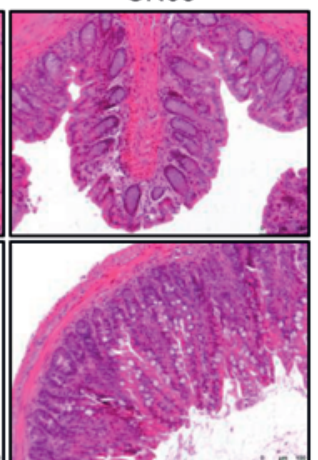

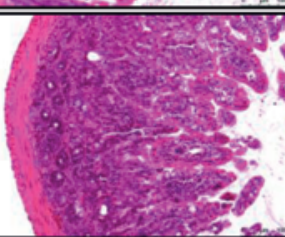

$\mathrm{CNO} 3$

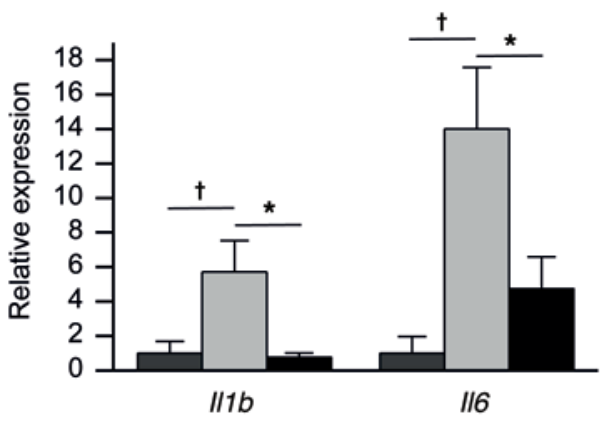

B

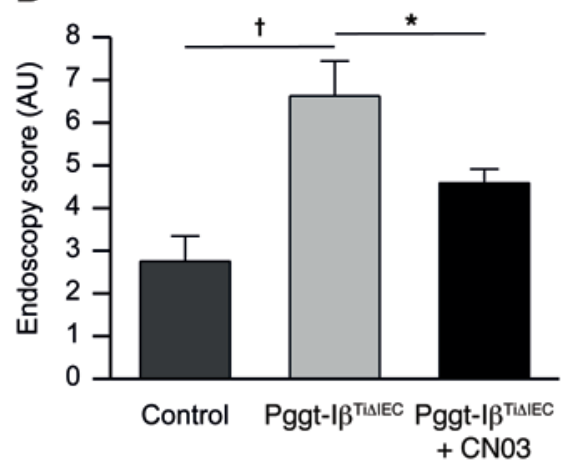

D

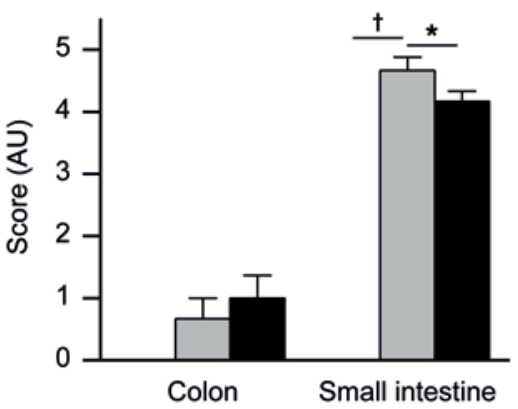

CN03

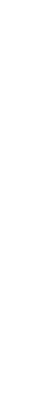

Figure 14. Treatment of Pggt-I $\boldsymbol{\beta}^{\text {TiAlEc }}$ mice with the Rho activator CN03. Three independent experiments. (A) Representative colonic endoscopy pictures. (B) Endoscopy score. (C) Histologic analysis: representative pictures from H\&E staining. Original magnification, $\times 20$. (D) Damage score quantification. (E)

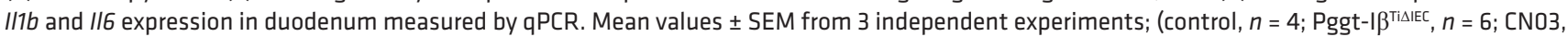
$n=6$ per group) in $\mathbf{B}, \mathbf{D}$, and $\mathbf{E}$. ${ }^{\dagger} P \leq 0.05$ vs. control mice; ${ }^{*} P \leq 0.05$ vs. Pggt-I $\beta^{\text {TislEc }}$ mice; 1 -way ANOVA with LSD multiple comparisons test in $\mathbf{B}$, $\mathbf{D}$, and $\mathbf{E}$.

Human samples. Human material was obtained from Department of Medicine I, University Clinic Erlangen, Erlangen, Germany; and Department of Gastroenterology, Humanitas Research Hospital, Milan, Italy. The collection of samples was approved by the local ethical committee and the institutional review board of the University of Erlangen-Nuremberg and Humanitas Research Hospital, and each patient gave written informed consent. Tissue samples were embedded in a convenient matrix for cryosectioning and frozen at $-80^{\circ} \mathrm{C}$ or freshly used for IEC isolation. Clinical information of patients included in our studies is summarized in Supplemental Tables 1-4.

Intestinal epithelial permeability assessment (FITC-Dextran). Intestinal permeability was assessed in vivo (51). In brief, Pggt-I $\beta^{\text {TiLIEC }}$ and control mice were deprived of food and water for 4 hours. Afterward, 60 mg FITC-Dextran (Sigma-Aldrich)/100 g body weight was given by oral gavage $(4,000 \mathrm{~g} / \mathrm{mol}$ average molecular weight of FITC-Dextran). Serum was collected 4 hours after gavage, and FITC concentration was determined spectrophotometrically using FITC-Dextran as standard.

In vivo cell shedding. In short $(5,27)$, mice were anesthetized by ketamin/xylazin i.p. injection, and the intestine was exteriorized and opened. The mucosa was topically stained with acriflavine (SigmaAldrich) and rhodamine-dextran $(10,000 \mathrm{~g} / \mathrm{mol})$ as luminal dye (Invitrogen). Surgical preparation was pinned up on a coverslide and mounted in a chamber for perfusion of saline solution. Time-sequential Z-stacks images of the villus tip were taken with a confocal microscope (Leica Microsystems).

Quantification of epithelial cell shedding. Epithelial cell shedding was quantified by several techniques. On one side, the cell shedding rate was quantified by the counting of cell shedding events detected by 
in vivo cell imaging. The number of cell shedding events was normalized by the length of the basal membrane on the individual pictures and the time of image acquisition. A total number of 10 animals were analyzed. On the other hand, we have also counted the number of leakage and "permeable cells" (our terminology for cells with dextran in the cytoplasm) in 2 single in vivo images from each specimen $(n=8$ animals). Finally, a similar method was performed in order to quantify the number of arrested versus completed cell shedding events. However, in this case, we took advantage of actin staining with phalloidin, which allowed us to detect the cytoskeleton protein redistribution occurring in early stages of the cell shedding process. We thus quantified the overall cell shedding rate, the distribution of different stages of cell shedding, and the appearance of dextran-permeable cells.

IEC isolation. Murine gut tissues and human surgical specimens were freshly used for IEC isolation. Therefore, tissue was incubated in a solution containing DTT and EDTA (34). Purification of IECs was performed by Percoll gradient.

Crypt isolation and organoid culture. Small intestine was aseptically isolated, washed, and cut. Intestinal fragments were washed, incubated in chelation buffer (containing EDTA), and vigorously resuspended in order to isolate intestinal crypts. Crypts were embedded in Matrigel (BD Biosciences) on ice and seeded in surface cell culture plates. After polymerization of Matrigel, basal culture medium was added to the culture (24). The entire medium was changed every 2 days, and organoids were passaged weekly. Organoids were treated in vitro with $0.5 \mu \mathrm{g} / \mathrm{ml}$ tamoxifen; the caspase inhibitor Z-VAD (10 $\mu \mathrm{g} / \mathrm{ml}$ ) (Bachem) or Necrostatin-1 (30 $\mu \mathrm{M})$ (Sigma-Aldrich), and cytokines $(20 \mathrm{ng} / \mathrm{ml})$.

Quantification of cell viability and death in crypt organoid cultures. We used a cell viability imaging kit (Roche Diagnostics) in order to quantify the death/destruction of organoids after in vitro treatment of organoids with tamoxifen and subsequent deletion of Pggt1b gene. This kit includes 3 different dyes in order to identify nuclei (blue dye), viable cells (green dye), and dead cells (red dye). Identification of dead cells is based on permeability of those cells due to destruction of the cell membrane. Detection of cell viability is based on metabolic activity, which allows the cleavage of the substrate, resulting in green fluorescence. Nuclear staining (blue) enabled normalization of results based on the overall number of detected cells. Quantification of the mean intensity of dead cell signal (red signal) and nuclear signal (blue signal) was performed using ImageJ software.

Histology, IHC, and electron microscopy. Formalin-fixed paraffin-embedded samples were used for H\&E staining and histopathological analysis. The score of the histological damage in colon, ileum, and duodenum took into account the 2 parameters epithelial erosion and distorted tissue architecture. Each parameter was scored (none, 0 ; mild, 1; pronounced, 2; severe, 3), and both results were summed afterward (maximum score of 6). Scoring was performed in a blinded fashion. IHC was performed in cryosections, using TSA Cy3 (PerkinElmer). Images were acquired by fluorescence microscopy (Olympus or Leica) or by confocal microscopy (Leica TCS SP5 or Leica TCS SP8). The following primary antibodies were used overnight at $4^{\circ} \mathrm{C}$ : GGTase-I $\beta, 1: 100$ (catalog sc-18996, Santa Cruz Biotechnology Inc.); Np-Rap1A, 1:100 (catalog sc-1482, Santa Cruz Biotechnology Inc.; for exclusive detection of Np-Rap1A); EpCAM, 1:100 (catalogs 324201 and 118207, BioLegend); MPO, 1:250 (catalog ab9535, Abcam); CD4, 1:200 (catalog 100506, BioLegend); F4/80, 1:100 (catalog 123101,
BioLegend); CD8 $\alpha, 1: 200$ (catalog 14-0081, eBioscience); Ki67, 1:1,000 (catalog ab16667, Abcam); Myosin-IIA, 1:200 (catalog 3403, Cell Signaling Technology); cleaved caspase-3, 1:750 (catalog AF835, R\&D Systems); and Rho-A, 1:100 (catalogs sc-418 and sc-179, Santa Cruz Biotechnology Inc.). Biotinylated-Streptavidin antibody pairs were used for detection (1:100-1:200, 30 minutes at room temperature (RT), Santa Cruz Biotechnology Inc., BioLegend). In some cases, direct dye-labeled secondary antibodies were used (1:100, 30 minutes at RT, BioLegend). Actin fiber staining was performed using phalloidin (Invitrogen). In situ cell death detection kit (Roche Diagnostics) was used for detection of TUNEL ${ }^{+}$cells. For electron microscopy, glutaraldehyde-fixed material was used. After embedding in Epon Araldite, ultrathin sections were cut and analyzed using a Zeiss EM 906.

Quantification of IHC. We have exclusively measured the average intensity of GGTase-I $\beta$ or Rap1A signal from epithelium. Using ImageJ software, $\mathrm{EpCAM}^{+}$cells (IECs) were selected, the mean intensity of epithelial GGTase-I $\beta$ or Rap1A signal was detected, and the overall number of IECs was counted based on nuclear staining. The overall number of IECs per image was used for normalization. Using this strategy, we analyzed 2 representative images per experiment in $n=4$ experiments. Overall, 8 images from each group (control and inflamed) were included into data interpretation.

The number of $\mathrm{CD}^{+}, \mathrm{F} 4 / 80^{+}, \mathrm{MPO}^{+}$, and $\mathrm{CD} 8^{+}$cells per field was counted using ImageJ software. A total of 3 different images/sample ( $\times 20$ objective) were counted, and the average cell number was calculated accordingly.

RNA isolation and quantitative PCR ( $P$ PCR) analysis. Total RNA was isolated from tissues or IECs using RNeasy Mini kit (QIAGEN). Synthesis of cDNA was performed using reverse transcriptase. Gene expression was measured by real-time PCR (Bio-Rad) with SYBR Green and QuantiTect primers (QIAGEN). Gene expression was normalized to the housekeeping gene HPRT expression.

Gene expression assay (mouse; array data accessible through through NCBI's GEO [GSE72781]). Gene-chip experiments were performed by the Erlangen University core facility ( $n=3$ per group). RNA quality was first assessed by Eukaryotic Total RNA Nano (Agilent Technologies), and gene expression was measured using the Affymetrix mouse 4302.0 chip. For multiple gene array testing, including differential expression analysis, the software package FlexArray (Michal Blazejczyk, Mathieu Miron, and Robert Nadon [2007], Genome Quebec, Montreal, Canada; http://www.gqinnovationcenter.com/services/ bioinformatics/index.aspx?l=e) was used. GO-based analyses were performed using the online tool Database for Annotation, Visualization, and Integrated Discovery (DAVID) and the platform Ingenuity.

Gene expression assay (human; array data accessible through through NCBI's GEO [GSE72780]). More than 30 samples were collected from the gut of patients suffering from CD or control patients. IECs were isolated from patient specimen as described. RNA was isolated using the RNeasy Lipid Tissue Mini kit according to manufacturer's instructions (QIAGEN). Nine samples were chosen for GeneChip analysis according to the following inclusion criteria: histopathological confirmation of inflammation status, acceptable RNA quality as confirmed by a bioanalyzer, and comparable expression of the epithelial marker villin.

GeneChip analysis. Genome-wide expression analysis was performed in the Genomics core facility of the Institute of Human Genetics of the University Erlangen-Nuremberg using the Affymetrix GeneChip Human Genome U133 Plis 2.0 Arrays. For multiple gene 
array evaluations, including advanced significance analysis, clustering, and gene ontology-based gene class testings, ANOVA of the software package Partek Genomics Suite were used. In brief, GC-RMA algorithm was applied to CEL-files of all microarrays. Next, log scale transformation, grouping, and baseline definition were performed. For advanced significance analysis, the following parameters were applied: unpaired $t$ test, $P<0.05$, fold-change $>1.5$, K-Means Clustering. Redundancies in gene list (e.g., cluster probe test lists) were eliminated, and gene ontology enrichment analysis was performed, allowing the detection of statistically overrepresented protein functions (GO terms) in a subset of genes. More than 54,000 expression tags were analyzed, from which 129 significantly $(P<0.05)$ differed (fold-change $>1.5$ ) between IECs from inflamed and uninflamed gut areas of CD patients.

Immunoblotting. Protein extracts were obtained by suspension and incubation in mammalian protein extraction reagent (Thermo Scientific) supplemented with protease and phosphatase inhibitor tablets (Roche Diagnostics). For membrane/cytosol fraction separation, lysis buffer (RIPA) with and without detergents and high-speed centrifugation steps were used. Protein extract was cleared by centrifugation, protein concentration was measured by Bradford assays, and denaturation was performed by boiling in LDS sample buffer (Thermo Scientific). Afterward, proteins were separated by SDSPAGE gels, blotted onto nitrocellulose or polyvinylidene fluoride (PVDF) membranes, and blocked with 5\% nonfat milk. Membranes were incubated overnight at $4^{\circ} \mathrm{C}$ with the following primary antibodies: GGTase-I $\beta$, 1:2,500 (catalog WH0005229M2, Sigma-Aldrich); Np-Rap1A, 1:1,000 (catalog sc-1482, Santa Cruz Biotechnology Inc.); Rho-A, 1:500 (catalog sc-179, Santa Cruz Biotechnology Inc.); phospho-MLC2, 1:1,000 (catalog 3671, Cell Signaling Technology); Cdc42, 1:1,000 (catalog 2466, Cell Signaling Technology); Rac-1, 1:1,000 (catalog sc-95, Santa Cruz Biotechnology Inc.); Rho-B, 1:1,000 (cata$\log$ 2098, Cell Signaling Technology); Rho-C, 1:1,000 (catalog 3430, Cell Signaling Technology); and AHRR, 1:1,000 (catalog sc-138745, Santa Cruz Biotechnology Inc.). Convenient HRP-linked secondary antibodies were used (incubation for 90 minutes at RT), and chemoluminescence was detected by using ECL Western blotting substrate (Thermo Scientific). Protein expression was compared with the level of actin, 1:2,000 (catalog 4967, Cell Signaling Technology). Inclusion of a positive control sample (protein lysate from testis) allowed reliable identification of the 38-43 kDa protein GGTase-I.

Proteomic assay. Of the total protein from purified IECs, $20 \mu \mathrm{g}$ were digested using a modified filter aided sample preparation (FASP) method (52). Three biological replicates were included, except where indicated. Reversed-phase nano-UPLC separation of tryptic peptides and mass spectrometric analysis using an ion-mobility enhanced data-independent analysis workflow were performed as previously described (52). All samples were analyzed in 3 technical replicates.

Cytoskeleton protein activation array. Activation of 64 cytoskeleton-related proteins was assessed by measuring their phosphorylation status using Cytoskeleton II Phospho Antibody Array (catalog PCP141, Cytoskeleton Inc.), following manufacturer's instructions.
Rho-A activation assay. Rho-A activation status was measured by using Rho-A G-LISA Activation Assay (catalog BK214, Cytoskeleton Inc.), following manufacturer's instructions.

Rho activator treatment. Mice were randomly distributed into 2 groups (Pggt-I $\beta^{\mathrm{TiAIEC}} \pm \mathrm{CNO} 3$ ) and Pggt-I ${ }^{\mathrm{flx}}$ littermates were used as controls. Mice were treated with the Rho Activyator II (catalog CNO3, Cytoskeleton Inc.) before onset of intestinal disease (oral, $0.3 \mu \mathrm{g} / \mathrm{mouse}$; intrarectal, $0.5 \mu \mathrm{g} / \mathrm{mouse}$ ). Tamoxifen was administered intrarectally. Animals were sacrificed 10 days after starting tamoxifen induction.

Statistics. Significance analysis of normally distributed data was performed using 2-tailed Student's $t$ test. Error bars represent \pm SEM. For multiple comparisons, data were analyzed by 1-way ANOVA and LSD post-hoc test (IBM SPSS Statistic 21). $P$ values of less than 0.05 and 0.001 were considered significant and highly significant.

Study approval. Animal protocols were approved by the Institutional Animal Care and Use Committee of the University of Erlangen. Animal studies were performed in accordance with German law and with approval from Regierung Mittelfranken (Ansbach, Germany). Collection of human samples was approved by Ethics Committee of the Department of Medicine of the Univeristy of Erlangen-Nuremberg (Erlangen, Germany) and Humanitas Research Hospital. Each patient gave written informed consent.

\section{Author contributions}

RLP, C Becker, IA, and MFN designed the research. RLP and VT performed the majority of the experiments. CG performed gene expression analysis in human IECs. ST performed quantitative proteomic analysis. KA performed electron microscopy. UB, RA, GF, SV, and SD collected human material for the study. AJMW was involved in performance of live animal imaging. SW was involved in discussion and design of experiments. $\mathrm{C}$ Brakebusch and $\mathrm{MB}$ provided us with genetically modified mouse strains. RLP, IA, MFN, AJMW, and C Becker analyzed the data and wrote the paper. $\mathrm{ABE}$ performed Gene-chip experiments. All the authors discussed the results and commented on the manuscript.

\section{Acknowledgments}

The research leading to these results has received funding from the People Programme (Marie Curie Actions) under Research Executive Agency of the European Commission (REA) grant agreement number 302170 and from the IMI Initiative (BTCure) of the European Union's Seventh Framework Programme (FP7/2007-2013). The work was funded by the Interdisciplinary Center for Clinical Research (IZKF) of the University Erlangen-Nuremberg. The German Research Foundation DFG supported this work within priority program SPP1656 (Intestinal Microbiota) and the clinical research unit $\mathrm{KFO} 257$.

Address correspondence to: Imke Atreya, Translational Research Center (TRC), Schwabachanlage 12, 91054 Erlangen, Germany. Phone: 49.0.9131.85.39602; E-mail: imke.atreya@ uk-erlangen.de.
1. Turner JR. Intestinal mucosal barrier function in health and disease. Nat Rev Immunol. 2009;9(11):799-809.

2. van der Flier LG, Clevers H. Stem cells, self- renewal, and differentiation in the intestinal epithelium. Annu Rev Physiol. 2009;71:241-260.

3. Watson AJ, Duckworth CA, Guan Y, Montrose MH. Mechanisms of epithelial cell shedding in the Mammalian intestine and maintenance of barrier function. Ann N Y Acad Sci. 2009;1165:135-142.

4. Gunther C, et al. Caspase-8 regulates TNF- $\alpha$ - 
induced epithelial necroptosis and terminal ileitis. Nature. 2011;477(7364):335-339.

5. Watson AJ, et al. Epithelial barrier function in vivo is sustained despite gaps in epithelial layers. Gastroenterology. 2005;129(3):902-912.

6. Pastorelli L, De Salvo C, Mercado JR, Vecchi M, Pizarro TT. Central role of the gut epithelial barrier in the pathogenesis of chronic intestinal inflammation: lessons learned from animal models and human genetics. Front Immunol. 2013;4:280.

7. Maloy KJ, Powrie F. Intestinal homeostasis and its breakdown in inflammatory bowel disease. Nature. 2011;474(7351):298-306.

8. Kiesslich R, et al. Local barrier dysfunction identified by confocal laser endomicroscopy predicts relapse in inflammatory bowel disease. Gut. 2012;61(8):1146-1153.

9. Ray RM, Vaidya RJ, Johnson LR. MEK/ERK regulates adherens junctions and migration through Rac1. Cell Motil Cytoskeleton. 2007;64(3):143-156.

10. Melendez J, et al. Cdc42 coordinates proliferation, polarity, migration, and differentiation of small intestinal epithelial cells in mice. Gastroenterology. 2013;145(4):808-819.

11. Citalan-Madrid AF, Garcia-Ponce A, Vargas-Robles H, Betanzos A, Schnoor M. Small GTPases of the Ras superfamily regulate intestinal epithelial homeostasis and barrier function via common and unique mechanisms. Tissue Barriers. 2013;1(5):e26938.

12. Benoit $Y D$, et al. Integrin $\alpha 8 \beta 1$ regulates adhesion, migration and proliferation of human intestinal crypt cells via a predominant RhoA/ ROCK-dependent mechanism. Biol Cell. 2009;101(12):695-708.

13. Hopkins AM, Walsh SV, Verkade P, Boquet P, Nusrat A. Constitutive activation of Rho proteins by CNF-1 influences tight junction structure and epithelial barrier function. J Cell Sci. 2003;116(pt 4):725-742.

14. Olofsson B. Rho guanine dissociation inhibitors: pivotal molecules in cellular signalling. Cell Signal. 1999;11(8):545-554.

15. Ridley AJ. Rho GTPases and actin dynamics in membrane protrusions and vesicle trafficking. Trends Cell Biol. 2006;16(10):522-529.

16. Adamson P, Paterson HF, Hall A. Intracellular localization of the P21rho proteins. JCell Biol. 1992;119(3):617-627.

17. Zverina EA, Lamphear CL, Wright EN, Fierke CA. Recent advances in protein prenyltransferases: substrate identification, regulation, and disease interventions. Curr Opin Chem Biol. 2012;16(5-6):544-552.

18. Eisenhoffer GT, et al. Crowding induces live cell extrusion to maintain homeostatic cell numbers in epithelia. Nature. 2012;484(7395):546-549.

19. Slattum G, McGee KM, Rosenblatt J. P115 RhoGEF and microtubules decide the direction apoptotic cells extrude from an epithelium. JCell Biol. 2009;186(5):693-702.

20. Reed RA, et al. Morphogenesis of the primitive gut tube is generated by Rho/ROCK/myosin IImediated endoderm rearrangements. Dev Dyn. 2009;238(12):3111-3125.

21. Jackson B, et al. RhoA is dispensable for skin development, but crucial for contraction and directed migration of keratinocytes. Mol Biol Cell. 2011;22(5):593-605.

22. el Marjou F, et al. Tissue-specific and inducible Cre-mediated recombination in the gut epithelium. Genesis. 2004;39(3):186-193.

23. Pechlivanis M, Kuhlmann J. Hydrophobic modifications of Ras proteins by isoprenoid groups and fatty acids - more than just membrane anchoring Biochim Biophys Acta. 2006;1764(12):1914-1931.

24. Sato T, et al. Single Lgr5 stem cells build cryptvillus structures in vitro without a mesenchymal niche. Nature. 2009;459(7244):262-265.

25. Drastich P, Frolova-Brizova L, Zanvit P, Spicak J, Tlaskalova-Hogenova H. Spontaneous in vitro IL-6 production in various intestinal segments in patients with inflammatory bowel disease. Folia Microbiol (Praha). 2011;56(3):185-190.

26. Sjogren AK, et al. GGTase-I deficiency reduces tumor formation and improves survival in mice with K-RAS-induced lung cancer. JClin Invest. 2007;117(5):1294-1304

27. Marchiando AM, et al. The epithelial barrier is maintained by in vivo tight junction expansion during pathologic intestinal epithelial shedding. Gastroenterology. 2011;140(4):1208-1218 e1201.

28. Turcotte JF, et al. Increased epithelial gaps in the small intestine are predictive of hospitalization and surgery in patients with inflammatory bowel disease. Clin Transl Gastroenterol. 2012;3:e19.

29. Lim LG, et al. Confocal endomicroscopy identifies loss of local barrier function in the duodenum of patients with Crohn's disease and ulcerative colitis. Inflamm Bowel Dis. 2014;20(5):892-900.

30. Debant A, et al. The multidomain protein Trio binds the LAR transmembrane tyrosine phosphatase, contains a protein kinase domain, and has separate rac-specific and rho-specific guanine nucleotide exchange factor domains. Proc Natl Acad Sci U S A. 1996;93(11):5466-5471.

31. Monteleone I, Pallone F, Monteleone G. Aryl hydrocarbon receptor and colitis. Semin Immunopathol. 2013;35(6):671-675.

32. Hahn ME, Allan LL, Sherr DH. Regulation of constitutive and inducible AHR signaling: complex interactions involving the AHR repressor. Biochem Pharmacol. 2009;77(4):485-497.

33. Ravi A, Garg P, Sitaraman SV. Matrix metalloproteinases in inflammatory bowel disease: boon or a bane? Inflamm Bowel Dis. 2007;13(1):97-107.

34. Nenci A, et al. Epithelial NEMO links innate immunity to chronic intestinal inflammation. Nature. 2007;446(7135):557-561.

35. Heller F, et al. Interleukin-13 is the key effector Th2 cytokine in ulcerative colitis that affects epithelial tight junctions, apoptosis, and cell restitution. Gastroenterology. 2005;129(2):550-564.

36. Braegger CP, Nicholls S, Murch SH, Stephens S, MacDonald TT. Tumour necrosis factor $\alpha$ in stool as a marker of intestinal inflammation. Lancet. 1992;339(8785):89-91.

37. Al-Sadi R, et al. Interleukin- 6 modulation of intestinal epithelial tight junction permeability is mediated by JNK pathway activation of claudin-2 gene. PLoS One. 2014;9(3):e85345

38. Watson AJ, Hughes KR. TNF- $\alpha$-induced intes- tinal epithelial cell shedding: implications for intestinal barrier function. Ann N Y Acad Sci. 2012;1258:1-8.

39. Coffelt SB, et al. The pro-inflammatory peptide LL-37 promotes ovarian tumor progression through recruitment of multipotent mesenchymal stromal cells. Proc Natl Acad Sci U S A. 2009;106(10):3806-3811.

40. Ma TY, et al. TNF-alpha-induced increase in intestinal epithelial tight junction permeability requires NF-KB activation. Am J Physiol Gastrointest Liver Physiol. 2004;286(3):G367-G376.

41. Fries $\mathrm{W}$, et al. Intestinal permeability and genetic determinants in patients, first-degree relatives, and controls in a high-incidence area of Crohn's disease in Southern Italy. Am J Gastroenterol. 2005;100(12):2730-2736.

42. Terc J, Hansen A, Alston L, Hirota SA. Pregnane $\mathrm{X}$ receptor agonists enhance intestinal epithelial wound healing and repair of the intestinal barrier following the induction of experimental colitis. Eur J Pharm Sci. 2014;55:12-19.

43. Waschke J, Spindler V, Bruggeman P, Zillikens D, Schmidt G, Drenckhahn D. Inhibition of Rho A activity causes pemphigus skin blistering. JCell Biol. 2006;175(5):721-727.

44. May M, Wang T, Muller M, Genth H. Difference in F-actin depolymerization induced by toxin $\mathrm{B}$ from the Clostridium difficile strain VPI 10463 and toxin B from the variant Clostridium difficile serotype F strain 1470. Toxins (Basel). 2013;5(1):106-119.

45. Lin HJ, et al. Curcumin blocks migration and invasion of mouse-rat hybrid retina ganglion cells (N18) through the inhibition of MMP-2, -9, FAK, Rho A and Rock-1 gene expression. Oncol Rep. 2010;23(3):665-670.

46. Newton K, Sun X, Dixit VM. Kinase RIP3 is dispensable for normal NF-kBs, signaling by the $\mathrm{B}$-cell and T-cell receptors, tumor necrosis factor receptor 1, and Toll-like receptors 2 and 4. Mol Cell Biol. 2004;24(4):1464-1469.

47. Kitajima S, Takuma S, Morimoto M. Tissue distribution of dextran sulfate sodium (DSS) in the acute phase of murine DSS-induced colitis. JVet Med Sci. 1999;61(1):67-70.

48. Ohkusa T. [Production of experimental ulcerative colitis in hamsters by dextran sulfate sodium and changes in intestinal microflora]. Nihon Shoka kibyo Gakkai Zasshi.1985;82(5):1327-1336.

49. Becker C, et al. In vivo imaging of colitis and colon cancer development in mice using high resolution chromoendoscopy. Gut. 2005;54(7):950-954.

50. Becker C, Fantini MC, Neurath MF. High resolution colonoscopy in live mice. Nat Protoc. 2006;1(6):2900-2904

51. Yan Y, Dalmasso G, Nguyen HT, Obertone TS, Sitaraman SV, Merlin D. Ste20-related proline/ alanine-rich kinase (SPAK) regulated transcriptionally by hyperosmolarity is involved in intestinal barrier function. PLoS One. 2009;4(4):e5049.

52. Distler U, Kuharev J, Navarro P, Levin Y, Schild H, Tenzer S. Drift time-specific collision energies enable deep-coverage data-independent acquisition proteomics. Nat Methods. 2014;11(2):167-170 